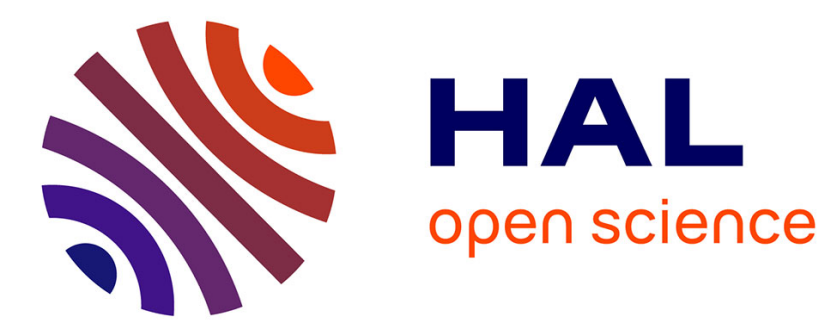

\title{
Micromechanics of elastoplastic porous polycrystals: theory, algorithm, and application to osteonal bone
}

\author{
Claire Morin, Viktoria Vass, Christian Hellmich
}

\section{To cite this version:}

Claire Morin, Viktoria Vass, Christian Hellmich. Micromechanics of elastoplastic porous polycrystals: theory, algorithm, and application to osteonal bone. International Journal of Plasticity, 2017. hal01671779

\section{HAL Id: hal-01671779 \\ https://hal.science/hal-01671779}

Submitted on 22 Dec 2017

HAL is a multi-disciplinary open access archive for the deposit and dissemination of scientific research documents, whether they are published or not. The documents may come from teaching and research institutions in France or abroad, or from public or private research centers.
L'archive ouverte pluridisciplinaire HAL, est destinée au dépôt et à la diffusion de documents scientifiques de niveau recherche, publiés ou non, émanant des établissements d'enseignement et de recherche français ou étrangers, des laboratoires publics ou privés. 


\title{
Micromechanics of elastoplastic porous polycrystals: theory, algorithm, and application to osteonal bone
}

\author{
Claire Morin ${ }^{\mathrm{a}, \mathrm{b}, \mathrm{c}, \mathrm{d}}$, Viktoria Vass ${ }^{\mathrm{a}}$, Christian Hellmich ${ }^{\mathrm{a}, *}$ \\ ${ }^{a}$ Institute for Mechanics of Materials and Structures, \\ TU Wien - Vienna University of Technology, A-1040 Vienna, Austria \\ ${ }^{b}$ Ecole Nationale Supérieure des Mines de Saint Etienne, \\ CIS-EMSE, SAINBIOSE, F-42023 Saint-Etienne, France \\ ${ }^{c}$ INSERM U1059, SAINBIOSE, F-42023 Saint-Etienne, France \\ ${ }^{d}$ Université de Lyon, F-69000 Lyon, France
}

\begin{abstract}
Since its advent in the 1960s, elastoplastic micromechanics has been confronted by continuous challenges, as the classical incremental elastoplastic tangents are known to deliver unrealistically stiff material responses. As a complement to the various "secant" approximations targeting this challenge, we here develop a theoretical framework based on an extension of Dvorak's transformation field analysis, comprising the derivation of concentration and influence tensors. We thereby overcome the problem of actually non-homogeneous stress distributions across finite (often spherical) material phases, through consideration of infinitely many (non-spherical) solid phases oriented in all space directions, arriving at a micro-elastoplasticity theory of porous polycrystals. The resulting governing equations are discretized in time and space, and then solved in the framework of a new return mapping algorithm, the realization of which we exemplify by means of Mohr-Coulomb plasticity at the solid phase level. The CORRESPONDING HOMOGENIZED MATERIAL LAW IS FINALLY SHOWN TO SATISFACTORILY REPRESENT THE BEHAVIOR OF THE POROUS HYDROXYAPATITE POLYCRYSTALS MAKING UP THE SO-CALLED CEMENT LINES IN OSTEONAL BONE. THIS IS EXPERIMENTALLY VALIDATED THROUGH STRENGTH AND ULTRASONIC TESTS ON
\end{abstract}

\footnotetext{
${ }^{*}$ Corresponding author. Tel.: +4315880120220; fax: +4315880120299.

Email addresses: claire.morin@emse.fr (Claire Morin),

Viktoria.Vass@tuwien.ac at (Viktoria Vass), Christian.Hellmich@tuwien.ac.at (Christian Hellmich)
} 
HYDROXYAPATITE, AS WELL AS THROUGH LIGHT MICROSCOPY, CHEMICAL COMPOSITION, AND OSTEON PUSHOUT TESTS ON BONE.

Keywords: microstructures, elastic-plastic material, inhomogeneous material, polycrystalline material, numerical algorithms 


\section{Nomenclature}

\begin{tabular}{|c|c|}
\hline $\mathbb{A}_{i}$ & fourth-order strain concentration tensor of phase $i$ \\
\hline $\mathbb{A}_{\text {pore }}$ & fourth-order strain concentration tensor of pore phase \\
\hline $\mathbb{A}_{\text {pore }}^{\infty}$ & $\begin{array}{l}\text { matrix-inclusion problem-related strain concentration ten- } \\
\text { sor of the pore phase }\end{array}$ \\
\hline $\mathbb{A}_{\theta \phi}$ & $\begin{array}{l}\text { fourth-order strain concentration tensor of solid needle } \\
\text { phase with orientation }(\theta, \phi)\end{array}$ \\
\hline $\mathbb{A}_{\theta \phi}^{\infty}$ & $\begin{array}{l}\text { matrix-inclusion problem-related strain concentration ten- } \\
\text { sor of the solid needle phase oriented in direction }(\theta, \phi)\end{array}$ \\
\hline$c_{\text {solid }}$ & cohesion of solid phases \\
\hline $\mathbb{C}_{0}$ & stiffness tensor of matrix in matrix-inclusion problem \\
\hline $\mathbb{C}_{H A}$ & stiffness tensor of hydroxyapatite \\
\hline $\mathbb{C}_{\text {hom }}$ & homogenized stiffness tensor (of porous polycrystal) \\
\hline $\mathbb{C}_{\text {pore }}$ & stiffness tensor of pore phase \\
\hline $\mathbb{C}_{\text {solid }}$ & stiffness tensor of solid needle phases \\
\hline$d$ & $\begin{array}{l}\text { characteristic length of the inhomogeneities within the } \\
\text { RVE }\end{array}$ \\
\hline $\mathbb{D}_{i j}$ & $\begin{array}{l}\text { FOURTH-ORDER INFLUENCE TENSOR LINKING EIGEN- } \\
\text { STRAINS IN INTEGRATION POINT } j \text { TO TOTAL STRAINS } \\
\text { IN INTEGRATION POINT } i\end{array}$ \\
\hline $\mathbb{D}_{\text {pore }, \Theta \Phi}$ & $\begin{array}{l}\text { fourth-order influence tensor linking eigenstrains in }(\Theta, \Phi) \text { - } \\
\text { oriented solid needle phase to total strains in the pore phase }\end{array}$ \\
\hline $\mathbb{D}_{\theta \phi, \Theta \Phi}$ & $\begin{array}{l}\text { fourth-order influence tensor linking eigenstrains in }(\Theta, \Phi) \text { - } \\
\text { oriented solid needle phase to total strains in }(\theta, \phi) \text {-oriented } \\
\text { solid needle phase }\end{array}$ \\
\hline $\mathbb{D}_{\text {pore }, j}$ & $\begin{array}{l}\text { fourth-order influence tensor linking eigenstrains in phase } \\
j \text { to total strains in pore phase }\end{array}$ \\
\hline $\mathbf{E}$ & macroscopic strain tensor \\
\hline $\mathbf{E}^{n}, \mathbf{E}^{n+1}$ & $\begin{array}{l}\text { macroscopic strain tensor at load steps } n \text { and }(n+1) \text {, re- } \\
\text { spectively }\end{array}$ \\
\hline$\Delta \mathbf{E}^{n+1}$ & $(n+1)$-st macroscopic strain increment \\
\hline $\mathbf{E}_{0}$ & $\begin{array}{l}\text { homogeneous strains at the infinite boundary of the matrix- } \\
\text { inclusion problem }\end{array}$ \\
\hline $\mathbf{E}_{0}^{\pi}$ & strain-like quantity according to (33) \\
\hline $\mathbf{E}^{p}$ & macroscopic plastic strain tensor \\
\hline
\end{tabular}




\begin{tabular}{|c|c|}
\hline $\mathbf{E}^{p, n}, \mathbf{E}^{p, n+1}$ & $\begin{array}{l}\text { macroscopic plastic strains at load steps } n \text { and }(n+1) \text {, } \\
\text { respectively }\end{array}$ \\
\hline $\mathbf{e}_{1}, \mathbf{e}_{2}, \mathbf{e}_{3}$ & unit base vectors of Cartesian reference base frame \\
\hline $\mathbf{e}_{\theta}, \mathbf{e}_{\phi}, \mathbf{e}_{r}$ & $\begin{array}{l}\text { unit base vectors of spherical base frame attached to the } \\
\text { solid needle phase }\end{array}$ \\
\hline$f_{H A}$ & volume fraction of hydroxyapatite \\
\hline$f_{\text {pore }}$ & $\begin{array}{l}\text { volume fraction of (intercrystalline) pore space; i.e. (inter- } \\
\text { crystalline) porosity }\end{array}$ \\
\hline$f_{\text {vas }}$ & volume fraction of vascular pores; i.e. vascular porosity \\
\hline $\mathcal{F}^{(\alpha)}$ & $\alpha$-th yield function of the multisurface yield criterion \\
\hline $\mathcal{F}_{i}^{(\alpha), n+1, \text { trial }}$ & $\begin{array}{l}\text { trial state in phase } i \text { at load step }(n+1) \text {, of yield function } \\
\mathcal{F}^{(\alpha)}\end{array}$ \\
\hline $\mathcal{G}^{(\alpha)}$ & $\begin{array}{l}\text { plastic flow potential related to activity of the } \alpha \text {-th yield } \\
\text { function in multisurface yield criterion }\end{array}$ \\
\hline$i, j$ & INDICES NUMBERING INTEGRATION POINTS CHOSEN FOR \\
\hline & $\begin{array}{l}\text { EVALUATION OF INTEGRALS OVER THE UNIT SPHERE } \\
\text { (THE LATTER REPRESENTING THE SET OF ALL SPATIAL } \\
\text { ORIENTATIONS OF SOLID NEEDLE-SHAPED PHASES) }\end{array}$ \\
\hline $\mathbb{I}$ & fourth-order identity tensor \\
\hline $\mathbb{I}^{d e v}$ & deviatoric part of $\mathbb{I}$ \\
\hline $\mathbb{I}^{v o l}$ & volumetric part of $\mathbb{I}$ \\
\hline$k_{H A}$ & bulk modulus of hydroxyapatite \\
\hline$k_{\mathrm{H}_{2} \mathrm{O}}$ & bulk modulus of water \\
\hline$l$ & characteristic length of the RVE \\
\hline$l_{\theta \phi}$ & $\begin{array}{l}\text { length of all needle-shaped crystals oriented in }(\theta, \phi) \text { - } \\
\text { direction }\end{array}$ \\
\hline $\mathcal{L}$ & characteristic structural length \\
\hline $\mathbf{n}$ & unit normal vector at microscopic scale \\
\hline$N_{c y l, p l a s t}$ & $\begin{array}{l}\text { number of plasticizing needle-shaped (cylindrical) solid } \\
\text { phases }\end{array}$ \\
\hline$N_{\text {Fact }, i}$ & $\begin{array}{l}\text { number of active yield surfaces in (plasticizing) solid needle } \\
\text { phase } i\end{array}$ \\
\hline$N_{\text {Fact }, \text { max }}$ & $\begin{array}{l}\text { maximum number of potentially active yield surfaces per } \\
\text { solid needle phase }\end{array}$ \\
\hline$p_{I-I I}, p_{I-I I I}$ & $\begin{array}{l}\text { planes defining regions in stress space representations of } \\
\text { Mohr-Coulomb criterion }\end{array}$ \\
\hline $\mathbb{P}_{c y l}$ & $\begin{array}{l}\text { Hill (or morphology) tensor of cylindrical inclusion embed- } \\
\text { ded in matrix with stiffness } \mathbb{C}_{\text {hom }}\end{array}$ \\
\hline
\end{tabular}


$\mathbb{P}_{s p h}$

RVE

$\mathbf{R}^{n+1, k+1, l}$

$\mathcal{S}$

$\mathcal{S}_{\text {cyl }, \text { plast }}$ $t^{n}, t^{n+1}$

t

T

$V_{\text {pore }}$

$V_{R V E}$

$\partial V_{R V E}$

$W^{e x t}$

$W^{i n t}$

$\mathrm{x}$

$\beta$

$\delta$

$\varepsilon$

$\varepsilon_{i}$

$\varepsilon_{i}^{n}, \varepsilon_{i}^{n+1}$

$\varepsilon_{i}^{p}$

$\Delta \varepsilon_{i}^{p, n+1}$

$\varepsilon_{\text {pore }}$

$\varepsilon_{\text {pore }}^{n}, \varepsilon_{\text {pore }}^{n+1}$

$\varepsilon_{\theta \phi}$

$\varepsilon_{\theta \phi}^{p}$

Hill (or morphology) tensor of spherical inclusion embedded in matrix with stiffness $\mathbb{C}_{\text {hom }}$

Representative Volume Element

residual vector in Newton iteration scheme, related to load step $(n+1)$, to the $(k+1)$-st macro-micro transition, and to return mapping iteration $l$

set of Gaussian points over the unit sphere

set of plasticizing needle-shaped (cylindrical) solid phases time instants at load steps $n$ and $(n+1)$, respectively

vector orthogonal to $\mathbf{n}$, within the Mohr plane spanned by $\mathbf{n}$ and $\mathbf{T}$

(microscopic) traction vector

volume of pore phase

volume of the RVE

surface of the RVE

work of external forces acting on the RVE

work of internal forces acting within the RVE

position vector inside the RVE

ratio between uniaxial tensile strength and shear strength

of hydroxyapatite

Kronecker delta

microscopic strain

average microscopic strain in phase $i$

average microscopic strains in phase $i$, at load steps $n$ and $(n+1)$, respectively

average microscopic plastic strains in phase $i$

$(n+1)$-st increment of microscopic plastic strain tensor of phase $i$

average pore strains

average pore strains at load steps $n$ and $(n+1)$, respectively average microscopic strains in solid needle phase with orientation $(\theta, \phi)$

average plastic strains in solid needle phase with orientation $(\theta, \phi)$ 


\begin{tabular}{|c|c|}
\hline$\theta$ & spherical (co-latitudinal) coordinate \\
\hline$\dot{\lambda}_{\theta \phi}^{(\alpha)}$ & $\begin{array}{l}\text { plastic multiplier related to the } \alpha \text {-th yield surface associ- } \\
\text { ated to the solid needle phase oriented in }(\theta, \phi) \text {-direction }\end{array}$ \\
\hline$\Delta \boldsymbol{\lambda}^{n+1, k+1, l}$ & $\begin{array}{l}\text { plastic multiplier vector in Newton iteration scheme, re- } \\
\text { lated to load step }(n+1) \text {, to the }(k+1) \text {-st macro-micro } \\
\text { transition, and to return mapping iteration } l\end{array}$ \\
\hline$\Delta(\Delta \boldsymbol{\lambda})^{n+1, k+1, l}$ & $\begin{array}{l}\text { plastic multiplier vector update in Newton iteration } \\
\text { scheme, related to load step }(n+1) \text {, to the }(k+1) \text {-st macro- } \\
\text { micro transition, and to return mapping iteration } l\end{array}$ \\
\hline$\Delta \lambda_{i}^{(\alpha), n+1}$ & $\begin{array}{l}(n+1) \text {-st increment of plastic multiplier related to the } \alpha \text {-th } \\
\text { yield surface associated to phase } i\end{array}$ \\
\hline$\mu_{H A}$ & shear modulus of hydroxyapatite \\
\hline$\xi$ & microscopic displacement field \\
\hline $\boldsymbol{\pi}_{\theta \phi}$ & eigenstress of solid needle phase oriented in $(\theta, \phi)$-direction \\
\hline$\Pi^{0}$ & $\begin{array}{l}\text { eigenstress acting in the infinite matrix of the matrix- } \\
\text { inclusion problem }\end{array}$ \\
\hline$\rho_{e c}$ & extracellular bone tissue mass density \\
\hline$\rho_{\text {exvas }}$ & extravascular bone tissue mass density \\
\hline$\rho_{\text {macro }}$ & macroscopic mass density \\
\hline$\phi$ & spherical (longitudinal) coordinate \\
\hline$\varphi_{\text {solid }}$ & angle of internal friction \\
\hline$\sigma_{i, j}$ & ordered principal stresses of phase $i, j=I, I I, I I I$ \\
\hline$\sigma_{y, H A}$ & compressive yield strength of hydroxyapatite \\
\hline$\sigma$ & microscopic stress \\
\hline $\boldsymbol{\sigma}_{i}$ & average stress in phase $i$ \\
\hline $\boldsymbol{\sigma}_{i}^{n}, \boldsymbol{\sigma}_{i}^{n+1}$ & $\begin{array}{l}\text { average stress in phase } i \text { at load steps } n \text { and }(n+1) \text {, re- } \\
\text { spectively }\end{array}$ \\
\hline$\Delta \boldsymbol{\sigma}_{i}^{n+1}$ & $(n+1)$-st microscopic stress increment of phase $i$ \\
\hline $\boldsymbol{\sigma}_{i}^{n+1, t r i a l}$ & trial stress in phase $i$, at load step $(n+1)$ \\
\hline$\tilde{\boldsymbol{\sigma}}_{i}^{n+1}$ & $\begin{array}{l}\text { average stress in phase } i \text {, at load step }(n+1) \text {, as defined in } \\
(81)\end{array}$ \\
\hline$\sigma_{n n}=\sigma$ & normal component of traction vector \\
\hline$\sigma_{n t}=\tau$ & shear component of traction vector \\
\hline $\boldsymbol{\sigma}_{\text {pore }}$ & average pore stress \\
\hline $\boldsymbol{\sigma}_{\theta \phi}$ & average stress in solid needle phase with orientation $(\theta, \phi)$ \\
\hline
\end{tabular}


$\Sigma$

$\Sigma^{n}, \Sigma^{n+1}$

$\Delta \Sigma^{n+1}$

$\omega_{i}$

$\nabla^{s}$

$\langle()$.

div

$(.)^{T}$

$(.)^{-1}$

$\otimes$

(.) macroscopic stress tensor

macroscopic stress tensor at load steps $n$ and $(n+1)$, respectively

$(n+1)$-st macroscopic stress increment

Gaussian weight

symmetric gradient operator

spatial average of quantity (.), over the RVE

divergence operator

transpose of tensorial quantity (.)

inverse of tensorial quantity (.)

dyadic product

rate (temporal derivative) of quantity (.) 


\section{Introduction}

Homogenization theories for elastic properties, whose high maturity has been reviewed in many textbooks and review papers (Nemat-Nasser and Hori, 1999; Zaoui, 2002; Dormieux et al., 2006), have fundamentally shaped the understanding and design of microheterogeneous materials, such as metals (Mori and Tanaka, 1973), ceramics (Constantinides and Ulm, 2004), or hard biological materials (Fritsch and Hellmich, 2007). By comparison, the field of micro-elastic-plasticity, although emerging almost as early as the elasticity homogenization theories, namely in the 1960 s, seems to remain remarkably less influential on materials research and design. It appears that elastoplastic homogenization is much more tricky than its purely elastic counterpart. This becomes already obvious from a very compact historical review: Already in 1965, Hill proposed an incremental method (Hill, 1965a) for elastoplastic upscaling: The microscopic elastoplastic behavior is represented by a (timedependent) phase-specific elastoplastic tangent tensor which multilinearly relates microstresses to microstrains; and this formally "elastic" relation is then simply inserted into classical homogenization schemes for the realm of elasticity, such as the self-consistent method (Hershey, 1954; Kröner, 1958) or the Mori-Tanaka method (Mori and Tanaka, 1973; Benveniste, 1987). However, the results obtained by this approach appeared as consistently too stiff when compared to full elastoplastic Finite Element simulations of the microstructure - and this has, up to the present day, motivated a series of interesting correction schemes, such as "isotropization" of the (originally anisotropic) elastoplastic tangent (or secant) operators, see e.g. (Berveiller and Zaoui, 1978; Doghri and Ouaar, 2003; Chaboche et al., 2005; Shen et al., 2012; Cayzac et al., 2013; Rousselier and Luo, 2014; Shen and Shao, 2016) and the various references cited there.

It took until the early 1990s that Dvorak and coworkers proposed a more fundamental approach to the topic. Considering that plastic microstrains are, as of their nature, kinematically incompatible, and therefore qualify as free strains or eigenstrains, Dvorak and co-workers developed a new type of homogenization theory, called "transformation field analysis - TFA" (Dvorak and Benveniste, 1992; Dvorak, 1992; Dvorak et al., 1994). Corresponding homogenization schemes (which will be more deeply reviewed and described in Section 3) provide access to the so-called concentration tensors $\mathbb{A}_{i}$ and influence tensors $\mathbb{D}_{i j}$, which relate the microscopic strains in the $i$-th material phase, $\boldsymbol{\varepsilon}_{i}$, to the macroscopic strains $\mathbf{E}$ prescribed at the boundary of 
the Representative Volume Element (RVE) and to all the free (here plastic) strains $\varepsilon_{j}^{p}$ occurring in all the other material phases,

$$
\varepsilon_{i}=\mathbb{A}_{i}: \mathbf{E}+\sum_{j} \mathbb{D}_{i j}: \varepsilon_{j}^{p}
$$

Derivation of (1) with respect to time readily delivers

$$
\dot{\varepsilon}_{i}=\mathbb{A}_{i}: \dot{\mathbf{E}}+\sum_{j} \mathbb{D}_{i j}: \dot{\varepsilon}_{j}^{p}
$$

It is instructive to note that relation (2) is fundamentally different from the standard concentration relation in elastic homogenization, which for the case of elastoplastic tangent upscaling according to Hill's incremental method would read as (Hill, 1965b; Zaoui, 2002)

$$
\dot{\varepsilon}_{i}=\mathbb{A}_{i}\left(\mathbb{C}_{i}=\mathbb{C}_{i}^{e p s}\right): \dot{\mathbf{E}}
$$

Comparison of (3) and (2) highlights that the use of the elastic concentration (or localization) problem for upscaling elastoplastic tangent tensors $\mathbb{C}_{i}^{\text {eps }}$ (rather than elasticity tensors $\mathbb{C}_{i}$ ) obviously neglects several sources for microscopic deformations. This is consistent with the repeatedly made statement that the incremental homogenization method delivers results which are too stiff. Accordingly, very promising examples for the use of (1) and (2), with properties derived from unit cell methods, could be given for periodic microstructures by Dvorak et al. (1994); Kruch and Chaboche (2011); Cavalcante and Pindera (2016). However, in case of more complex and more random micromorphologies, the proper choice and number of phases (with uniform plastic strains), which would actually allow for appropriately covering the complex plastic flow patterns across the microstructures, often appears as the real (and hard-to-master) challenge when applying the "classical" TFA. While the introduction of "plastic modes" across material phases [in the course of non-uniform TFA - NTFA (Michel and Suquet, 2003, 2004; Roussette et al., 2009; Fritzen and Böhlke, 2011)] showed interesting ways to overcome the aforementioned problem for various applications, we here follow yet another approach, which proved recently very successful in the context of elastic, of poroelastic, and of brittle strength upscaling; across various material classes such as hydroxyapatite, bioactive glass ceramics, gypsum, cementitious materials, and piezoelectric ceramics (Fritsch et al., 2006, 2009a,b, 
2013; Pichler et al., 2008; Pichler and Hellmich, 2011; Pichler et al., 2013). In this approach, the representative volume element is divided into infinitely many non-spherical solid phases oriented in all space directions, and spherical pores in-between. It is for this micromechanical representation, that we here develop an elastoplastic homogenization theory based on a recent extension and generalization of the transformation field analysis (Dvorak et al., 1994). This is described in the remainder of the present paper: In Section 2, the concept of the representative volume element is reviewed in the framework of the principle of virtual power (Germain, 1973; Salençon, 2001), and then specified for a porous polycrystalline material system built up by elastoplastic needle-shaped solid elements and pores in-between. Thereafter, upscaling of elastoplastic behavior from the level of the solid needles to that of the overall polycrystalline material is covered in Section 3, by introducing phasespecific concentration, influence, as well as homogenized stiffness tensors. Semi-analytical expressions for the latter are derived in Section 4, based on eigenstressed matrix-inclusion problems of the Eshelby-Laws type. The resulting governing equations are then discretized in time and space, and solved in the framework of a return mapping algorithm described in Section 5; the realization of which we exemplify by means of Mohr-Coulomb plasticity at the solid phase level in Section 6. Finally, the new theory and computational model is applied to osteoneal bone, in Section 7; followed by Conclusions in Section 8.

\section{Representative volume element (RVE) - momentum balance and kinematic compatibility - microstructural characteristics of porous polycrystals}

The investigated material is considered as a macro-homogeneous but micro-heterogeneous matter filling a Representative Volume Element (RVE) (Hill, 1963; Zaoui, 2002). Such RVEs fulfill the separation of scales requirement:

$$
d \ll l \ll \mathcal{L}
$$

with $d$ as the characteristic length of the inhomogeneities within the RVE, $l$ as the characteristic length of the RVE, and $\mathcal{L}$ as the characteristic size of the body or structure made up by the material, or of loads acting on this body, such as wavelengths in case of dynamic loading. We consider an RVE subjected to homogeneous linearized macroscopic strains prescribed in terms 
of displacements

$$
\text { on } \partial V_{R V E} \quad \boldsymbol{\xi}(\mathbf{x})=\mathbf{E} \cdot \mathbf{x}
$$

with $\mathbf{x}$ as the location vector labeling points within the RVE and at its boundary. Homogeneous boundary conditions (5) and compatibility of microstrains within the RVE

$$
\forall \mathbf{x} \in V_{R V E} \quad \boldsymbol{\varepsilon}(\mathbf{x})=\nabla^{S} \boldsymbol{\xi}(\mathbf{x})
$$

directly imply the so-called strain average rule (Hashin, 1983)

$$
\frac{1}{V_{R V E}} \int_{V_{R V E}} \varepsilon(\mathbf{x}) d V=\langle\varepsilon\rangle=\mathbf{E}
$$

Furthermore, the aforementioned deformations provoke traction forces $\mathbf{T}(\mathbf{x})$ on the boundary of the RVE, and microstresses $\boldsymbol{\sigma}(\mathbf{x})$ throughout the RVE, fulfilling the equilibrium conditions

$$
\begin{aligned}
& \forall \mathbf{x} \in V_{R V E} \quad \operatorname{div} \boldsymbol{\sigma}(\mathbf{x})=0 \\
& \forall \mathbf{x} \in \partial V_{R V E} \quad \mathbf{T}(\mathbf{x})=\boldsymbol{\sigma}(\mathbf{x}) \cdot \mathbf{n}(\mathbf{x})
\end{aligned}
$$

with $\mathbf{n}(\mathbf{x})$ as the normal to the boundary at position $\mathbf{x}$. The external work done by these traction forces reads as

$$
\begin{aligned}
W^{e x t} & =\int_{\partial V_{R V E}} \mathbf{T}(\mathbf{x}) \cdot \boldsymbol{\xi}(\mathbf{x}) d S=\int_{\partial V_{R V E}}(\mathbf{E} \cdot \mathbf{x}) \cdot[\boldsymbol{\sigma}(\mathbf{x}) \cdot \mathbf{n}(\mathbf{x})] d S \\
& =\mathbf{E}: \int_{V_{R V E}} \boldsymbol{\sigma}(\mathbf{x}) d V
\end{aligned}
$$

Hence, the force quantity doing work on the macroscopic strains $\mathbf{E}$ is the volume integral over the microscopic stress, which is independent of microscopic position and dimension "stress times volume". This induces the existence of the macroscopic stress $\Sigma$ in the form

$$
\boldsymbol{\Sigma} V_{R V E}=\int_{V_{R V E}} \boldsymbol{\sigma}(\mathbf{x}) d V \Leftrightarrow \boldsymbol{\Sigma}=\frac{1}{V_{R V E}} \int_{V_{R V E}} \boldsymbol{\sigma}(\mathbf{x}) d V=\langle\boldsymbol{\sigma}\rangle,
$$

i.e. the well-known stress average rule. Insertion of (10) into the principle of virtual power (Germain, 1973; Salençon, 2001), which in the case of linearized strains, can be expressed in terms of an expression with the dimension "work",

$$
W^{e x t}=-W^{i n t}=\frac{1}{V_{R V E}} \int_{V_{R V E}} \boldsymbol{\sigma}(\mathbf{x}): \boldsymbol{\varepsilon}(\mathbf{x}) d V=\langle\boldsymbol{\sigma}: \boldsymbol{\varepsilon}\rangle
$$




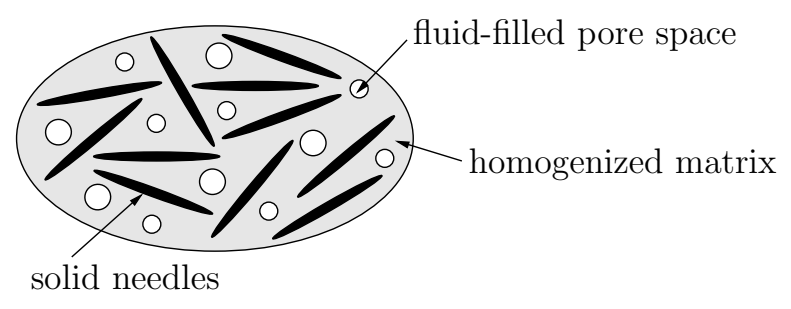

Figure 1: Representative Volume Element of the porous polycrystal

yields the so-called Hill's lemma

$$
\boldsymbol{\Sigma}: \mathbf{E}=\frac{1}{V_{R V E}} \int_{V_{R V E}} \boldsymbol{\sigma}(\mathbf{x}): \boldsymbol{\varepsilon}(\mathbf{x}) d V .
$$

As the microstructure cannot be described in complete detail, the morphological description is restricted to mechanically relevant features, through introduction of subdomains within the RVE, called phases. The latter exhibit homogeneous mechanical properties as described in further detail in Section 3. The mechanical state of these phases is characterized by stress and strain averages. For the present case of a porous polycrystal as depicted in Figure 1 , a "pore phase" fills subvolume $V_{\text {pore }}$ within the RVE, with respective stress and strain averages following from

$$
\begin{aligned}
& \boldsymbol{\sigma}_{\text {pore }}=\frac{1}{V_{\text {pore }}} \int_{V_{\text {pore }}} \boldsymbol{\sigma}(\mathbf{x}) d \mathbf{x} \\
& \boldsymbol{\varepsilon}_{\text {pore }}=\frac{1}{V_{\text {pore }}} \int_{V_{\text {pore }}} \boldsymbol{\varepsilon}(\mathbf{x}) d \mathbf{x}
\end{aligned}
$$

The rest of the RVE is filled by elongated solid crystal phases which are fully characterized by the orientation in space, through orientation vector $\mathbf{e}_{r}$ (see Figure 2): The latter can be given as a function of an orthonormal base frame $\mathbf{e}_{1}, \mathbf{e}_{2}$, and $\mathbf{e}_{3}$, and of longitudinal and co-latitudinal angles $\phi$ and $\theta$,

$$
\boldsymbol{e}_{r}=\sin \theta\left(\cos \phi \boldsymbol{e}_{1}+\sin \phi \boldsymbol{e}_{2}\right)+\cos \theta \boldsymbol{e}_{3}
$$


The corresponding stress and strain averages depend on the aforementioned angles, and read as

$$
\begin{aligned}
& \boldsymbol{\sigma}_{\theta \phi}=\frac{1}{l_{\theta \phi}} \int_{l_{\theta \phi}} \boldsymbol{\sigma}(s) d s \\
& \boldsymbol{\varepsilon}_{\theta \phi}=\frac{1}{l_{\theta \phi}} \int_{l_{\theta \phi}} \boldsymbol{\varepsilon}(s) d s
\end{aligned}
$$

with $l_{\theta \phi}$ as the length of all needle-shaped crystals oriented in $(\theta, \phi)$-direction. As a result, the strain average rules can be given in the following form, respectively

$$
\begin{gathered}
\boldsymbol{\Sigma}=f_{\text {pore }} \boldsymbol{\sigma}_{\text {pore }}+\left(1-f_{\text {pore }}\right) \int_{\theta=0}^{\pi} \int_{\phi=0}^{2 \pi} \boldsymbol{\sigma}_{\theta \phi} \frac{\sin \theta}{4 \pi} d \theta d \phi \\
\boldsymbol{E}=f_{\text {pore }} \boldsymbol{\varepsilon}_{\text {pore }}+\left(1-f_{\text {pore }}\right) \int_{\theta=0}^{\pi} \int_{\phi=0}^{2 \pi} \boldsymbol{\varepsilon}_{\theta \phi} \frac{\sin \theta}{4 \pi} d \theta d \phi
\end{gathered}
$$

whereby $f_{\text {pore }}=V_{\text {pore }} / V_{R V E}$ is the volume fraction of the pore space (i.e. the porosity).

\section{Elastoplasticity of solid phases - upscaling to porous polycrystal scale}

The following constitutive laws are assigned to the material phases depicted in Figure 1: In order to keep the mathematical descriptions of our developments as concise as possible, the spherical pore phase is simply characterized by a linear elastic material behavior

$$
\boldsymbol{\sigma}_{\text {pore }}=\mathbb{C}_{\text {pore }}: \boldsymbol{\varepsilon}_{\text {pore }}
$$

with $\mathbb{C}_{\text {pore }}$ as the fourth-order isotropic stiffness tensor of the pore space. In case of drained conditions, $\mathbb{C}_{\text {pore }}$ may be typically set to zero; and the extension to a full poromechanical formulation (Dormieux et al., 2006) may be realized through the introduction of additional eigenstresses representing pore pressures (Pichler and Hellmich, 2010; Fritsch et al., 2013). The needleshaped solid phases exhibit an elastic-perfectly plastic behavior

$$
\boldsymbol{\sigma}_{\theta \phi}=\mathbb{C}_{\text {solid }}:\left[\varepsilon_{\theta \phi}-\varepsilon_{\theta \phi}^{p}\right]
$$




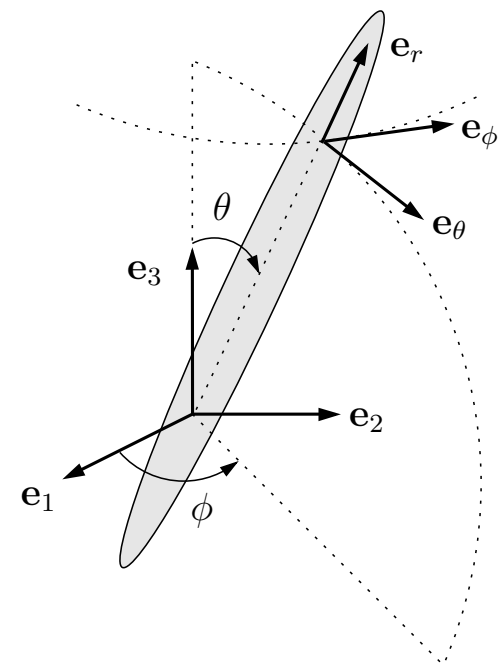

Figure 2: Orientation of solid needle by means of spherical coordinates $\theta$ and $\phi$, and definition of corresponding spherical base frame

with $\mathbb{C}_{\text {solid }}$ as their (isotropic) fourth-order stiffness tensor and $\varepsilon_{\theta \phi}^{p}$ as the average plastic strain in the solid NEEDLE phase oriented in $(\theta, \phi)$-direction. These plastic strains follow the multisurface plasticity flow rule according to Koiter (1953)

$$
\dot{\varepsilon}_{\theta \phi}^{p}=\sum_{\alpha=1}^{N_{F a c t, m a x}} \dot{\lambda}_{\theta \phi}^{(\alpha)} \frac{\partial \mathcal{G}^{(\alpha)}\left(\boldsymbol{\sigma}_{\theta \phi}\right)}{\partial \boldsymbol{\sigma}_{\theta \phi}}
$$

and occurrence of these strains is governed by the Melan-Kuhn-Tucker conditions

$$
\begin{array}{ll} 
& \dot{\lambda}_{\theta \phi}^{(\alpha)} \geq 0 \\
\forall \alpha \in\left\{1, \ldots, N_{\text {Fact,max }}\right\} \quad & \mathcal{F}^{(\alpha)}\left(\boldsymbol{\sigma}_{\theta \phi}\right) \leq 0 \\
& \dot{\lambda}_{\theta \phi}^{(\alpha)} \times \mathcal{F}^{(\alpha)}\left(\boldsymbol{\sigma}_{\theta \phi}\right)=0
\end{array}
$$

with $N_{\text {Fact,max }}$ as the total number of yield surfaces $\mathcal{F}^{(\alpha)}$ and of plastic potentials $\mathcal{G}^{(\alpha)}$ defining the multisurface yield criteria and flow rules; with associated plastic multipliers $\lambda_{\theta \phi}^{(\alpha)}$, governing the magnitude of the plastic strain rate.

For a linear elastic medium with eigenstrains, the latter (in our case, the plastic strains $\varepsilon_{\theta \phi}^{p}$ ) are related to the macroscopic strains through the so-called 
concentration-influence relations (Dvorak and Benveniste, 1992; Pichler and Hellmich, 2010); reading in the present case for the solid needle phases as

$$
\varepsilon_{\theta \phi}=\mathbb{A}_{\theta \phi}: \boldsymbol{E}+\int_{\Theta=0}^{\pi} \int_{\Phi=0}^{2 \pi} \mathbb{D}_{\theta \phi ; \Theta \Phi}: \varepsilon_{\Theta \Phi}^{p} \frac{\sin \Theta}{4 \pi} d \Theta d \Phi
$$

and for the pores

$$
\varepsilon_{\text {pore }}=\mathbb{A}_{\text {pore }}: \boldsymbol{E}+\int_{\Theta=0}^{\pi} \int_{\Phi=0}^{2 \pi} \mathbb{D}_{\text {pore } ; \Theta \Phi}: \varepsilon_{\Theta \Phi}^{p} \frac{\sin \Theta}{4 \pi} d \Theta d \Phi
$$

In these relations, $\mathbb{A}_{\theta \phi}$ and $\mathbb{A}_{\text {pore }}$ are the strain concentration tensors in the needle-shaped particles with orientation $(\theta, \phi)$ and in the SPHERICAL pore phase respectively; $\mathbb{D}_{\theta \phi ; \Theta \Phi}$ is the influence tensor expressing the effect of the plastic strain in the solid needles with orientation $(\Theta, \Phi)$ on the overall strain in the solid needles with orientation $(\theta, \phi) ; \mathbb{D}_{\text {pore } ; \Theta \Phi}$ is the influence tensor expressing the effect of the plastic strain in the solid needles with orientation $(\Theta, \Phi)$ on the overall strain in the pore phase.

The stress and strain average rules allow for upscaling the microscopic constitutive law to the macroscopic scale. In the presence of eigenstrains inside the RVE, the homogenized constitutive law is accessed through Levin's theorem (Levin, 1967; Laws, 1973)

$$
\boldsymbol{\Sigma}=\mathbb{C}_{\text {hom }}:\left(\boldsymbol{E}-\boldsymbol{E}^{p}\right)
$$

with $\mathbb{C}_{\text {hom }}$ as the homogenized stiffness tensor (Zaoui, 2002), defined by

$$
\begin{aligned}
\mathbb{C}_{\text {hom }} & =\langle\mathbb{C}: \mathbb{A}\rangle_{V_{R V E}}=f_{\text {pore }} \mathbb{C}_{\text {pore }}: \mathbb{A}_{\text {pore }} \\
& +\left(1-f_{\text {pore }}\right) \mathbb{C}_{\text {solid }}: \int_{\theta=0}^{\pi} \int_{\phi=0}^{2 \pi} \mathbb{A}_{\theta \phi} \frac{\sin \theta}{4 \pi} d \theta d \phi
\end{aligned}
$$

and with $\boldsymbol{E}^{p}$ as the macroscopic "plastic" strain, reading as (Levin, 1967; Laws, 1973; Zaoui, 2002)

$$
\begin{aligned}
\boldsymbol{E}^{p} & =\left\langle\varepsilon^{p}: \mathbb{C}: \mathbb{A}: \mathbb{C}_{\text {hom }}^{-1}\right\rangle \\
& =\left(1-f_{\text {pore }}\right) \mathbb{C}_{\text {hom }}^{-1}: \int_{\theta=0}^{\pi} \int_{\phi=0}^{2 \pi} \mathbb{A}_{\theta \phi}^{T}: \mathbb{C}_{\text {solid }}: \varepsilon_{\theta \phi}^{p} \frac{\sin \theta}{4 \pi} d \theta d \phi
\end{aligned}
$$




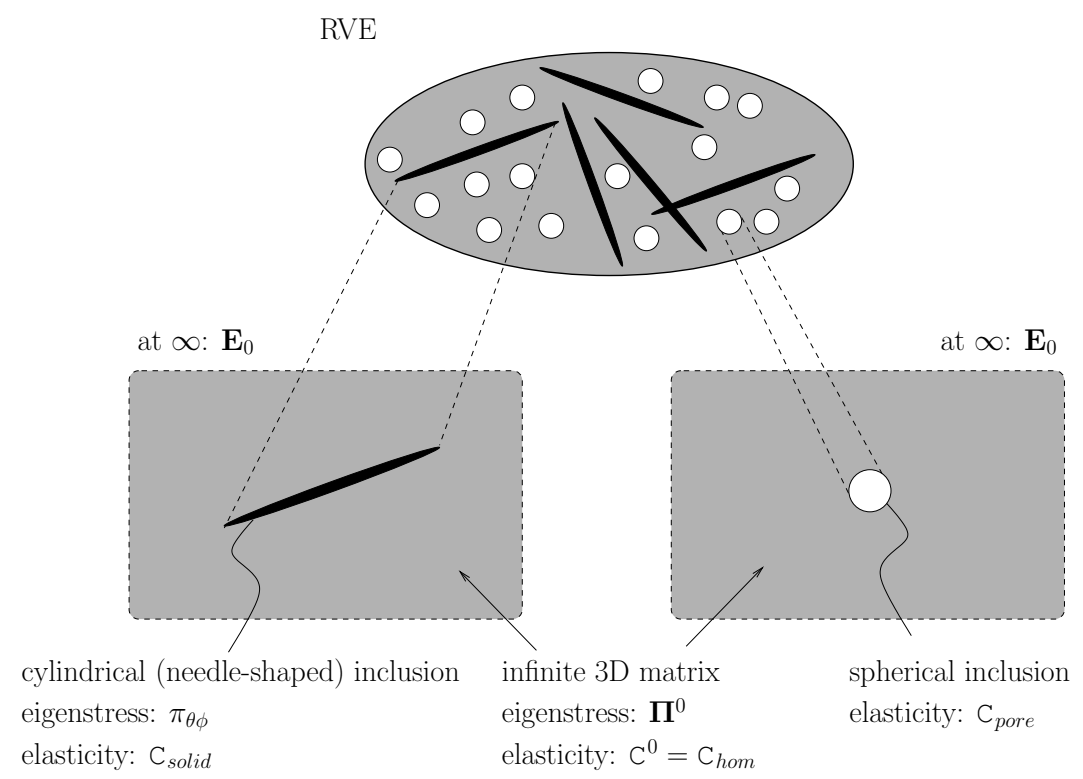

Figure 3: Quantifying RVE behavior through generalized Eshelby matrix-inclusion problems with eigenstresses

\section{Self-consistent estimation of concentration and influence tensors from eigenstressed Eshelby problems}

Self-consistent estimates for the strain concentration and influence tensors appearing in (22) and (23) are obtained by means of generalized Eshelby matrix-inclusion-type problems (Pichler and Hellmich, 2010; Zaoui, 2002). The pore phase is represented as a spherical inclusion embedded in a matrix with stiffness $\mathbb{C}_{\text {hom }}$ and eigenstress $\boldsymbol{\Pi}^{0}$, subjected to homogeneous strains $\boldsymbol{E}_{0}$ acting at the infinite boundary of the aforementioned matrix, see Figure 3. The needle-shaped solid phases are represented each as a cylindrical inclusion with stiffness $\mathbb{C}_{\text {solid }}$ and eigenstresses $\boldsymbol{\pi}_{\theta \phi}=-\mathbb{C}_{\text {solid }}: \varepsilon_{\theta \phi}^{p}$, embedded into the very same matrix, and subjected to the very same strains $\boldsymbol{E}_{0}$, see Figure 3. This results to inclusion/phase strains reading as (Zaoui, 2002)

$$
\begin{gathered}
\boldsymbol{\varepsilon}_{\theta \phi}=\mathbb{A}_{\theta \phi}^{\infty}:\left[\boldsymbol{E}_{0}-\mathbb{P}_{c y l}(\theta, \phi):\left(\boldsymbol{\pi}_{\theta \phi}-\boldsymbol{\Pi}^{0}\right)\right] \\
\boldsymbol{\varepsilon}_{\text {pore }}=\mathbb{A}_{\text {pore }}^{\infty}:\left[\boldsymbol{E}_{0}-\mathbb{P}_{\text {sph }}:\left(-\boldsymbol{\Pi}^{0}\right)\right]
\end{gathered}
$$

with the abbreviations $\mathbb{A}_{\theta \phi}^{\infty}$ and $\mathbb{A}_{\text {pore }}^{\infty}$ standing for

$$
\mathbb{A}_{\theta \phi}^{\infty}=\left[\mathbb{I}+\mathbb{P}_{\text {cyl }}(\theta, \phi):\left(\mathbb{C}_{\text {solid }}-\mathbb{C}_{\text {hom }}\right)\right]^{-1}
$$


and

$$
\mathbb{A}_{\text {pore }}^{\infty}=\left[\mathbb{I}+\mathbb{P}_{\text {sph }}:\left(\mathbb{C}_{\text {pore }}-\mathbb{C}_{\text {hom }}\right)\right]^{-1}
$$

whereby $\mathbb{I}$ is the fourth-order identity tensor, with components $I_{i j k l}=\frac{1}{2}\left(\delta_{i k} \delta_{j l}+\right.$ $\left.\delta_{i l} \delta_{j k}\right), \delta_{i j}$ is the Kronecker delta: $\delta_{i j}=1$ if $i=j$ and zero otherwise; $\mathbb{P}_{c y l}$ and $\mathbb{P}_{\text {sph }}$, respectively, are the Hill tensors of cylindrical and spherical inclusions embedded into a matrix with stiffness $\mathbb{C}_{\text {hom }}$. Insertion of (27) and (28) into the strain average rule (17) delivers

$$
\begin{aligned}
\boldsymbol{E} & =\left\{f_{\text {pore }} \mathbb{A}_{\text {pore }}^{\infty}:\left[\boldsymbol{E}_{0}-\mathbb{P}_{\text {sph }}:\left(-\boldsymbol{\Pi}^{0}\right)\right]\right. \\
& \left.+\left(1-f_{\text {pore }}\right) \int_{\theta=0}^{\pi} \int_{\phi=0}^{2 \pi} \mathbb{A}_{\theta \phi}^{\infty}:\left[\boldsymbol{E}_{0}-\mathbb{P}_{\text {cyl }}(\theta, \phi):\left(\boldsymbol{\pi}_{\theta \phi}-\boldsymbol{\Pi}^{0}\right)\right] \frac{\sin \theta}{4 \pi} d \theta d \phi\right\}
\end{aligned}
$$

Solving (31) for $\boldsymbol{E}_{0}$ yields

$$
\begin{aligned}
\boldsymbol{E}_{0} & =\left\{f_{\text {pore }} \mathbb{A}_{\text {pore }}^{\infty}\right. \\
& \left.+\left(1-f_{\text {pore }}\right) \int_{\theta=0}^{\pi} \int_{\phi=0}^{2 \pi} \mathbb{A}_{\theta \phi}^{\infty} \frac{\sin \theta}{4 \pi} d \theta d \phi\right\}^{-1}:\left\{\boldsymbol{E}+\boldsymbol{E}_{0}^{\pi}\right\}
\end{aligned}
$$

with

$$
\begin{aligned}
\boldsymbol{E}_{0}^{\pi} & =f_{\text {pore }} \mathbb{A}_{\text {pore }}^{\infty}: \mathbb{P}_{\text {sph }}:\left(-\boldsymbol{\Pi}^{0}\right) \\
& +\left(1-f_{\text {pore }}\right) \int_{\theta=0}^{\pi} \int_{\phi=0}^{2 \pi} \mathbb{A}_{\theta \phi}^{\infty}: \mathbb{P}_{c y l}(\theta, \phi):\left(\boldsymbol{\pi}_{\theta \phi}-\boldsymbol{\Pi}^{0}\right) \frac{\sin \theta}{4 \pi} d \theta d \phi
\end{aligned}
$$

Insertion of (32) into (27) and (28) yields

$$
\begin{gathered}
\boldsymbol{\varepsilon}_{\theta \phi}=\mathbb{A}_{\theta \phi}:\left\{\boldsymbol{E}+\boldsymbol{E}_{0}^{\pi}\right\}-\mathbb{A}_{\theta \phi}^{\infty}: \mathbb{P}_{c y l}(\theta, \phi):\left(\boldsymbol{\pi}_{\theta \phi}-\boldsymbol{\Pi}^{0}\right) \\
\boldsymbol{\varepsilon}_{\text {pore }}=\mathbb{A}_{\text {pore }}:\left\{\boldsymbol{E}+\boldsymbol{E}_{0}^{\pi}\right\}-\mathbb{A}_{\text {pore }}^{\infty}: \mathbb{P}_{\text {sph }}:\left(-\boldsymbol{\Pi}^{0}\right)
\end{gathered}
$$

with

$$
\begin{aligned}
& \mathbb{A}_{\theta \phi}=\mathbb{A}_{\theta \phi}^{\infty}:\left\{f_{\text {pore }} \mathbb{A}_{\text {pore }}^{\infty}+\left(1-f_{\text {pore }}\right) \int_{\Theta=0}^{\pi} \int_{\Phi=0}^{2 \pi} \mathbb{A}_{\Theta \Phi}^{\infty} \frac{\sin \Theta}{4 \pi} d \Theta d \Phi\right\}^{-1} \\
& \mathbb{A}_{\text {pore }}=\mathbb{A}_{\text {pore }}^{\infty}:\left\{f_{\text {pore }} \mathbb{A}_{\text {pore }}^{\infty}+\left(1-f_{\text {pore }}\right) \int_{\theta=0}^{\pi} \int_{\phi=0}^{2 \pi} \mathbb{A}_{\theta \phi}^{\infty} \frac{\sin \theta}{4 \pi} d \theta d \phi\right\}^{-1}
\end{aligned}
$$


Comparison of (34) and (35) with (22) and (23) makes readily evident that $\mathbb{A}_{\theta \phi}$ and $\mathbb{A}_{\text {pore }}$ are the sought expressions for the concentration tensors appearing in (22) and (23). As a second step, the stress average rule, combined with the Levin's theorem, allows for estimating the influence tensors. Therefore, (24) and (26) can be rewritten in the form,

$$
\begin{aligned}
\boldsymbol{\Sigma} & =\mathbb{C}_{\text {hom }}: \boldsymbol{E} \\
& +\left(1-f_{\text {pore }}\right) \int_{\theta=0}^{\pi} \int_{\phi=0}^{2 \pi} \boldsymbol{\pi}_{\theta \phi}: \mathbb{A}_{\theta \phi} \frac{\sin \theta}{4 \pi} d \theta d \phi
\end{aligned}
$$

On the other hand, the combination of the stress average rule (16) with constitutive equations (18) and (19), as well as with the scale transition relations (34) and (35), while considering the expression (25) for the homogenized stiffness, results in

$$
\begin{aligned}
\boldsymbol{\Sigma} & =f_{\text {pore }} \mathbb{C}_{\text {pore }}:\left\{\mathbb{A}_{\text {pore }}:\left\{\boldsymbol{E}+\boldsymbol{E}_{0}^{\pi}\right\}-\mathbb{A}_{\text {pore }}^{\infty}: \mathbb{P}_{\text {sph }}:\left(-\boldsymbol{\Pi}^{0}\right)\right\} \\
& +\left(1-f_{\text {pore }}\right) \int_{\theta=0}^{\pi} \int_{\phi=0}^{2 \pi}\left\{\mathbb{C}_{\text {solid }}:\left\{\mathbb{A}_{\theta \phi}:\left(\boldsymbol{E}+\boldsymbol{E}_{0}^{\pi}\right)\right.\right. \\
& \left.\left.-\mathbb{A}_{\theta \phi}^{\infty}: \mathbb{P}_{\text {cyl }}(\theta, \phi):\left(\boldsymbol{\pi}_{\theta \phi}-\boldsymbol{\Pi}^{0}\right)\right\}+\boldsymbol{\pi}_{\theta \phi}\right\} \frac{\sin \theta}{4 \pi} d \theta d \phi
\end{aligned}
$$

Setting the macroscopic stress relations (38) and (39) equal and solving the resulting expression for $\Pi^{0}$ delivers

$$
\begin{aligned}
\Pi^{0} & =\left\{\left(\mathbb{C}_{\text {hom }}-\mathbb{C}_{\text {pore }}\right): f_{\text {pore }} \mathbb{A}_{\text {pore }}^{\infty}: \mathbb{P}_{\text {sph }}\right. \\
& \left.+\left(\mathbb{C}_{\text {hom }}-\mathbb{C}_{\text {solid }}\right):\left(1-f_{\text {pore }}\right) \int_{\theta=0}^{\pi} \int_{\phi=0}^{2 \pi} \mathbb{A}_{\theta \phi}^{\infty}: \mathbb{P}_{\text {cyl }}(\theta, \phi) \frac{\sin \theta}{4 \pi} d \theta d \phi\right\}^{-1}: \\
& \left\{( 1 - f _ { \text { pore } } ) \int _ { \theta = 0 } ^ { \pi } \int _ { \phi = 0 } ^ { 2 \pi } \left[\boldsymbol{\pi}_{\theta \phi}:\left[\mathbb{I}-\mathbb{A}_{\theta \phi}\right]\right.\right. \\
& \left.\left.+\left(\mathbb{C}_{\text {hom }}-\mathbb{C}_{\text {solid }}\right): \mathbb{A}_{\theta \phi}^{\infty}: \mathbb{P}_{\text {cyl }}(\theta, \phi): \boldsymbol{\pi}_{\theta \phi}\right] \frac{\sin \theta}{4 \pi} d \theta d \phi\right\}
\end{aligned}
$$

Finally, inserting (40) into (34) and (35) and comparing the resulting expressions to (22) and (23) yield the eigenstress influence tensors $\mathbb{D}_{\text {pore }, \Theta \Phi}, \mathbb{D}_{\theta \phi, \Theta \Phi}$, 
and $\mathbb{D}_{\theta \phi, \theta \phi}$. They read as

$$
\begin{aligned}
& \mathbb{D}_{\text {pore }, \Theta \Phi}=\left\{-\mathbb{A}_{\text {pore }}\left(1-f_{\text {pore }}\right): \mathbb{A}_{\Theta \Phi}^{\infty}: \mathbb{P}_{\text {cyl }}(\Theta, \Phi)\right. \\
& +\left[\mathbb{A}_{\text {pore }} f_{\text {pore }}: \mathbb{A}_{\text {pore }}^{\infty}: \mathbb{P}_{\text {sph }}\right. \\
& +\mathbb{A}_{\text {pore }}\left(1-f_{\text {pore }}\right): \int_{\chi=0}^{\pi} \int_{\psi=0}^{2 \pi} \mathbb{A}_{\chi \psi}^{\infty}: \mathbb{P}_{c y l}(\chi, \psi) \frac{\sin \chi}{4 \pi} d \chi d \psi \\
& \left.-\mathbb{A}_{\text {pore }}^{\infty}: \mathbb{P}_{\text {sph }}\right]: \\
& {\left[\left(1-f_{\text {pore }}\right)\left(\mathbb{C}_{\text {hom }}-\mathbb{C}_{\text {solid }}\right): \int_{\chi=0}^{\pi} \int_{\psi=0}^{2 \pi} \mathbb{A}_{\chi \psi}^{\infty}: \mathbb{P}_{\text {cyl }}(\chi, \psi) \frac{\sin \chi}{4 \pi} d \chi d \psi\right.} \\
& \left.+f_{\text {pore }}\left(\mathbb{C}_{\text {hom }}-\mathbb{C}_{\text {pore }}\right): \mathbb{A}_{\text {pore }}^{\infty}: \mathbb{P}_{\text {sph }}\right]^{-1}: \\
& \left(1-f_{\text {pore }}\right)\left[\left[\mathbb{I}-\mathbb{A}_{\Theta \Phi}\right]^{T}\right. \\
& \left.+\left(\mathbb{C}_{\text {hom }}-\mathbb{C}_{\text {solid }}\right): \mathbb{A}_{\Theta \Phi}^{\infty}: \mathbb{P}_{\text {cyl }}(\Theta, \Phi)\right\}: \mathbb{C}_{\text {solid }} \\
& \mathbb{D}_{\theta \phi, \Theta \Phi}=\left\{-\mathbb{A}_{\theta \phi}:\left(1-f_{\text {pore }}\right) \mathbb{A}_{\Theta \Phi}^{\infty}: \mathbb{P}_{\text {cyl }}(\Theta, \Phi)\right. \\
& +\left[\mathbb{A}_{\theta \phi}:\left(1-f_{\text {pore }}\right) \int_{\chi=0}^{\pi} \int_{\psi=0}^{2 \pi} \mathbb{A}_{\chi \psi}^{\infty}: \mathbb{P}_{c y l}(\chi, \psi) \frac{\sin \chi}{4 \pi} d \chi d \psi\right. \\
& \left.+\mathbb{A}_{\theta \phi}: f_{\text {pore }}: \mathbb{A}_{\text {pore }}^{\infty}: \mathbb{P}_{\text {sph }}-\mathbb{A}_{\theta \phi}^{\infty}: \mathbb{P}_{\text {cyl }}(\theta, \phi)\right]: \\
& {\left[\left(1-f_{\text {pore }}\right)\left(\mathbb{C}_{\text {hom }}-\mathbb{C}_{\text {solid }}\right): \int_{\chi=0}^{\pi} \int_{\psi=0}^{2 \pi} \mathbb{A}_{\chi \psi}^{\infty}: \mathbb{P}_{\text {cyl }}(\chi, \psi) \frac{\sin \chi}{4 \pi} d \chi d \psi\right.} \\
& \left.+f_{\text {pore }}\left(\mathbb{C}_{\text {hom }}-\mathbb{C}_{\text {pore }}\right): \mathbb{A}_{\text {pore }}^{\infty}: \mathbb{P}_{\text {sph }}\right]^{-1}: \\
& \left(1-f_{\text {pore }}\right)\left[\left[\mathbb{I}-\mathbb{A}_{\Theta \Phi}\right]^{T}\right. \\
& \left.\left.+\left(\mathbb{C}_{\text {hom }}-\mathbb{C}_{\text {solid }}\right): \mathbb{A}_{\Theta \Phi}^{\infty}: \mathbb{P}_{\text {cyl }}(\Theta, \Phi)\right]\right\}: \mathbb{C}_{\text {solid }}
\end{aligned}
$$




$$
\begin{aligned}
\mathbb{D}_{\theta \phi, \theta \phi} & =\left\{\left[\mathbb{I}-\left(1-f_{\text {pore }}\right) \mathbb{A}_{\theta \phi}\right]: \mathbb{A}_{\theta \phi}^{\infty}: \mathbb{P}_{\text {cyl }}(\theta, \phi)\right. \\
& +\left[\mathbb{A}_{\theta \phi}:\left(1-f_{\text {pore }}\right) \int_{\chi=0}^{\pi} \int_{\psi=0}^{2 \pi} \mathbb{A}_{\chi \psi}^{\infty}: \mathbb{P}_{\text {cyl }}(\chi, \psi) \frac{\sin \chi}{4 \pi} d \chi d \psi\right. \\
& \left.+\mathbb{A}_{\theta \phi}: f_{\text {pore }} \mathbb{A}_{\text {pore }}^{\infty}: \mathbb{P}_{\text {sph }}-\mathbb{A}_{\theta \phi}^{\infty}: \mathbb{P}_{\text {cyl }}(\theta, \phi)\right]: \\
& {\left[\left(1-f_{\text {pore }}\right)\left(\mathbb{C}_{\text {hom }}-\mathbb{C}_{\text {solid }}\right): \int_{\chi=0}^{\pi} \int_{\psi=0}^{2 \pi} \mathbb{A}_{\chi \psi}^{\infty}: \mathbb{P}_{\text {cyl }}(\chi, \psi) \frac{\sin \chi}{4 \pi} d \chi d \psi\right.} \\
& \left.+f_{\text {pore }}\left(\mathbb{C}_{\text {hom }}-\mathbb{C}_{\text {pore }}\right): \mathbb{A}_{\text {pore }}^{\infty}: \mathbb{P}_{\text {sph }}\right]^{-1}: \\
& \left(1-f_{\text {pore }}\right)\left[\left[\mathbb{I}-\mathbb{A}_{\theta \phi}\right]^{T}\right. \\
& \left.\left.+\left(\mathbb{C}_{\text {hom }}-\mathbb{C}_{\text {solid }}\right): \mathbb{A}_{\theta \phi}^{\infty}: \mathbb{P}_{\text {cyl }}(\theta, \phi)\right]\right\}: \mathbb{C}_{\text {solid }}
\end{aligned}
$$

\section{Algorithmic treatment of multiscale elastoplasticity}

Numerical computations of plastic evolutions within the solid phases of the RVE depicted in Figure 1, as a function of arbitrary macroscopic loading in terms of macroscopic stresses $\boldsymbol{\Sigma}$ or macroscopic strains $\boldsymbol{E}$, requires temporal and spatial discretization of the elastoplastic relations (22)-(26), together with (41)-(43), and with average rules (16)-(17); as well as solution of the resulting algebraic equations. This will be dealt with in the following subsection.

\subsection{Spatial discretization of governing equations}

As regards spatial discretization, all integrals over the unit sphere are approximated through weighted sums of the integrands being evaluated at a particular set $\mathcal{S}$ of Gaussian points on the unit sphere (labelled by the two Euler angles $\left.\left\{\theta_{i}, \phi_{i}\right\}_{i \in \mathcal{S}}\right)$ with the associated Gaussian weights $\left.\omega_{i}\right|_{i \in \mathcal{S}}$. Accordingly, the integral over an arbitrary function $a(\Theta, \Phi)$ is approximated by the weighted sum over this function evaluated at particular Gaussian points labelled by index $i$; i.e. by the following discrete expression

$$
\iint_{\Theta, \Phi} a(\Theta, \Phi) \frac{\sin \Theta}{4 \pi} d \Theta d \Phi=\sum_{i \in \mathcal{S}} \omega_{i} a\left(\Theta_{i}, \Phi_{i}\right)=\sum_{i \in \mathcal{S}} \omega_{i} a_{i}
$$

This leads to the following discretized versions of the governing equations: 
- the (spatially discretized) stress and strain average rules

$$
\begin{gathered}
\boldsymbol{\Sigma}=f_{\text {pore }} \boldsymbol{\sigma}_{\text {pore }}+\sum_{i \in \mathcal{S}}\left(1-f_{\text {pore }}\right) \omega_{i} \boldsymbol{\sigma}_{i} \\
\boldsymbol{E}=f_{\text {pore }} \boldsymbol{\varepsilon}_{\text {pore }}+\sum_{i \in \mathcal{S}}\left(1-f_{\text {pore }}\right) \omega_{i} \boldsymbol{\varepsilon}_{i}
\end{gathered}
$$

- the (spatially discretized) concentration-influence relations

$$
\begin{gathered}
\forall i \in \mathcal{S} \quad \boldsymbol{\varepsilon}_{i}=\mathbb{A}_{i}: \boldsymbol{E}+\sum_{j \in \mathcal{S}} \omega_{j} \mathbb{D}_{i j}: \varepsilon_{j}^{p} \\
\varepsilon_{\text {pore }}=\mathbb{A}_{\text {pore }}: \boldsymbol{E}+\sum_{j \in \mathcal{S}} \omega_{j} \mathbb{D}_{\text {pore }, j}: \varepsilon_{j}^{p}
\end{gathered}
$$

whereby the discretized influence tensors $\mathbb{D}_{\text {pore }, j}, \mathbb{D}_{i j}$, and $\mathbb{D}_{i i}$ read as

$$
\begin{aligned}
\mathbb{D}_{\text {pore }, j}= & \left\{-\mathbb{A}_{\text {pore }}:\left(1-f_{\text {pore }}\right) \mathbb{A}_{j}^{\infty}: \mathbb{P}_{\text {cyl }, j}\right. \\
& +\left[f_{\text {pore }} \mathbb{A}_{\text {pore }}: \mathbb{A}_{\text {pore }}^{\infty}: \mathbb{P}_{\text {sph }}+\left(1-f_{\text {pore }}\right) \mathbb{A}_{\text {pore }}: \sum_{k \in \mathcal{S}} \omega_{k} \mathbb{A}_{k}^{\infty}: \mathbb{P}_{\text {cyl }, k}-\mathbb{A}_{\text {pore }}^{\infty}: \mathbb{P}_{\text {sph }}\right] \\
& :\left[f_{\text {pore }}\left(\mathbb{C}_{\text {hom }}-\mathbb{C}_{\text {pore }}\right): \mathbb{A}_{\text {pore }}^{\infty}: \mathbb{P}_{\text {sph }}\right. \\
& \left.+\left(1-f_{\text {pore }}\right)\left(\mathbb{C}_{\text {hom }}-\mathbb{C}_{\text {solid }}\right): \sum_{k \in \mathcal{S}} \omega_{k} \mathbb{A}_{k}^{\infty}: \mathbb{P}_{\text {cyl }, k}\right]^{-1} \\
& \left.:\left(1-f_{\text {pore }}\right)\left[\left(\mathbb{I}-\mathbb{A}_{j}\right)^{T}+\left(\mathbb{C}_{\text {hom }}-\mathbb{C}_{\text {solid }}\right): \mathbb{A}_{j}^{\infty}: \mathbb{P}_{\text {cyl }, j}\right]\right\}: \mathbb{C}_{\text {solid }}
\end{aligned}
$$




$$
\begin{aligned}
\mathbb{D}_{i j}= & \left\{-\mathbb{A}_{i}:\left(1-f_{\text {pore }}\right) \mathbb{A}_{j}^{\infty}: \mathbb{P}_{\text {cyl }, j}\right. \\
& +\left[f_{\text {pore }} \mathbb{A}_{i}: \mathbb{A}_{\text {pore }}^{\infty}: \mathbb{P}_{\text {sph }}+\left(1-f_{\text {pore }}\right) \mathbb{A}_{i}: \sum_{k \in \mathcal{S}} \omega_{k} \mathbb{A}_{k}^{\infty}: \mathbb{P}_{\text {cyl }, k}-\mathbb{A}_{i}^{\infty}: \mathbb{P}_{\text {cyl }, i}\right] \\
& :\left[f_{\text {pore }}\left(\mathbb{C}_{\text {hom }}-\mathbb{C}_{\text {pore }}\right): \mathbb{A}_{\text {pore }}^{\infty}: \mathbb{P}_{\text {sph }}\right. \\
& \left.+\left(1-f_{\text {pore }}\right)\left(\mathbb{C}_{\text {hom }}-\mathbb{C}_{\text {solid }}\right): \sum_{k \in \mathcal{S}} \omega_{k} \mathbb{A}_{k}^{\infty}: \mathbb{P}_{\text {cyl }, k}\right]^{-1} \\
& \left.:\left(1-f_{\text {pore }}\right)\left[\left(\mathbb{I}-\mathbb{A}_{j}\right)^{T}+\left(\mathbb{C}_{\text {hom }}-\mathbb{C}_{\text {solid }}\right): \mathbb{A}_{j}^{\infty}: \mathbb{P}_{\text {cyl }, j}\right]\right\}: \mathbb{C}_{\text {solid }}
\end{aligned}
$$$$
\mathbb{D}_{i i}=\left\{\left[\mathbb{I}-\left(1-f_{\text {pore }}\right) \mathbb{A}_{i}\right]: \mathbb{A}_{i}^{\infty}: \mathbb{P}_{c y l, i}\right.
$$$$
+\left[f_{\text {pore }} \mathbb{A}_{i}: \mathbb{A}_{\text {pore }}^{\infty}: \mathbb{P}_{\text {sph }}+\left(1-f_{\text {pore }}\right) \mathbb{A}_{i}: \sum_{k \in \mathcal{S}} \omega_{k} \mathbb{A}_{k}^{\infty}: \mathbb{P}_{\text {cyl }, k}-\mathbb{A}_{i}^{\infty}: \mathbb{P}_{c y l, i}\right]
$$$$
:\left[f_{\text {pore }}\left(\mathbb{C}_{\text {hom }}-\mathbb{C}_{\text {pore }}\right): \mathbb{A}_{\text {pore }}^{\infty}: \mathbb{P}_{\text {sph }}\right.
$$$$
\left.+\left(1-f_{\text {pore }}\right)\left(\mathbb{C}_{\text {hom }}-\mathbb{C}_{\text {solid }}\right): \sum_{k \in \mathcal{S}} \omega_{k} \mathbb{A}_{k}^{\infty}: \mathbb{P}_{\text {cyl }, k}\right]^{-1}
$$$$
\left.:\left(1-f_{\text {pore }}\right)\left[\left(\mathbb{I}-\mathbb{A}_{i}\right)^{T}+\left(\mathbb{C}_{\text {hom }}-\mathbb{C}_{\text {solid }}\right): \mathbb{A}_{i}^{\infty}: \mathbb{P}_{\text {cyl }, i}\right]\right\}: \mathbb{C}_{\text {solid }}
$$

- the microscopic state equation for the solid needle phases

$$
\forall i \in \mathcal{S} \quad \boldsymbol{\sigma}_{i}=\mathbb{C}_{\text {solid }}:\left(\varepsilon_{i}-\varepsilon_{i}^{p}\right)
$$

- the flow rule

$$
\forall i \in \mathcal{S} \quad \dot{\boldsymbol{\varepsilon}}_{i}^{p}=\sum_{\alpha=1}^{N_{\text {Fact,max }}} \dot{\lambda}_{i}^{(\alpha)} \frac{\partial \mathcal{G}^{(\alpha)}}{\partial \boldsymbol{\sigma}_{i}}\left(\boldsymbol{\sigma}_{i}\right)
$$

associated to the Melan-Kuhn-Tucker conditions

$$
\begin{aligned}
\forall \alpha \in\left\{1,2, \ldots, N_{\text {Fact }, \text { max }}\right\} & \dot{\lambda}_{i}^{(\alpha)} \geq 0 \\
& \mathcal{F}^{(\alpha)}\left(\boldsymbol{\sigma}_{i}\right) \leq 0 \\
& \dot{\lambda}_{i}^{(\alpha)} \times \mathcal{F}^{(\alpha)}\left(\boldsymbol{\sigma}_{i}\right)=0
\end{aligned}
$$


Table 1: 15 different needle orientations according to integration formulas of Stroud (1971)

\begin{tabular}{l|lllllllllllllll}
$j=$ & 1 & 2 & 3 & 4 & 5 & 6 & 7 & 8 & 9 & 10 & 11 & 12 & 13 & 14 & 15 \\
\hline $\sin \left(\theta_{j}\right) \cos \left(\phi_{j}\right)=$ & $+r$ & $+r$ & $-r$ & $-r$ & $+t$ & $+t$ & $-t$ & $-t$ & $+s$ & $+s$ & $-s$ & $-s$ & 1 & 0 & 0 \\
$\sin \left(\theta_{j}\right) \sin \left(\phi_{j}\right)=$ & $+s$ & $-s$ & $+s$ & $-s$ & $+r$ & $-r$ & $+r$ & $-r$ & $+t$ & $-t$ & $+t$ & $-t$ & 0 & 1 & 0 \\
$\cos \left(\theta_{j}\right)=$ & $+t$ & $+t$ & $+t$ & $+t$ & $+s$ & $+s$ & $+s$ & $+s$ & $+r$ & $+r$ & $+r$ & $+r$ & 0 & 0 & 1 \\
$\omega\left(\theta_{j}, \phi_{j}\right)=$ & $\frac{1}{15}$ & $\frac{1}{15}$ & $\frac{1}{15}$ & $\frac{1}{15}$ & $\frac{1}{15}$ & $\frac{1}{15}$ & $\frac{1}{15}$ & $\frac{1}{15}$ & $\frac{1}{15}$ & $\frac{1}{15}$ & $\frac{1}{15}$ & $\frac{1}{15}$ & $\frac{1}{15}$ & $\frac{1}{15}$ & $\frac{1}{15}$ \\
\hline & with & $r=1 / 2$, & $s=(\sqrt{5}+1) / 4$, & and & $t=(\sqrt{5}-1) / 4$ & & &
\end{tabular}

- the macroscopic (homogenized) stiffness tensor

$$
\mathbb{C}_{\text {hom }}=f_{\text {pore }} \mathbb{C}_{\text {pore }}: \mathbb{A}_{\text {pore }}+\left(1-f_{\text {pore }}\right) \sum_{i \in \mathcal{S}} \omega_{i} \mathbb{C}_{\text {solid }}: \mathbb{A}_{i}
$$

- and the macroscopic plastic strains

$$
\boldsymbol{E}^{p}=\left(1-f_{\text {pore }}\right) \mathbb{C}_{\text {hom }}^{-1}: \sum_{i \in \mathcal{S}} \omega_{i} \mathbb{A}_{i}^{T}: \mathbb{C}_{\text {solid }}: \varepsilon_{i}^{p}
$$

More precisely, we here employ two different sets $\mathcal{S}$ of Gaussian points:

- the Stroud's integration formulas (Stroud, 1971), comprising 15 or 28 points (see Tables 1 and 2)

- the centroids and areas of triangles meshing the unit sphere, as described in (Badel and Leblond, 2004). The triangular mesh of the unit sphere is obtained from the refinement of a semi-dodecahedron, splitting each pentagon into five triangles; further refinement can then be achieved by dividing each triangle into four smaller triangles. The centroids of the triangles are chosen as Gaussian points, while the ratio between the areas of the triangle and of the unit sphere is chosen as Gaussian weight. We use 120 integration points.

\subsection{Temporal discretization of governing equations}

As the spatially discretized temporal differential equations (45)-(56) cannot be solved analytically, approximative solutions will be obtained in an iterative process, which is described in greater detail in Sections 6 and 7 . This process relates to discrete time instants $t^{n}$ and corresponding stresses 
and strains, such as $E\left(t^{n}\right)=E^{n}$. Respective evaluations of (45)-(52) and (56) yield

$$
\begin{gathered}
\boldsymbol{\Sigma}^{n}=f_{\text {pore }} \boldsymbol{\sigma}_{\text {pore }}^{n}+\sum_{i \in \mathcal{S}}\left(1-f_{\text {pore }}\right) \omega_{i} \boldsymbol{\sigma}_{i}^{n} \\
\boldsymbol{E}^{n}=f_{\text {pore }} \varepsilon_{\text {pore }}^{n}+\sum_{i \in \mathcal{S}}\left(1-f_{\text {pore }}\right) \omega_{i} \varepsilon_{i}^{n} \\
\varepsilon_{i}^{n}=\mathbb{A}_{i}: \boldsymbol{E}^{n}+\sum_{j \in \mathcal{S}} \omega_{j} \mathbb{D}_{i j}: \varepsilon_{j}^{p, n} \\
\varepsilon_{\text {pore }}^{n}=\mathbb{A}_{\text {pore }}: \boldsymbol{E}^{n}+\sum_{i \in \mathcal{S}} \omega_{i} \mathbb{D}_{\text {pore } ; i}: \varepsilon_{i}^{p, n} \\
\boldsymbol{E}^{p, n}=\left(1-f_{\text {pore }}\right) \mathbb{C}_{\text {hom }}^{-1}: \sum_{i \in \mathcal{S}} \omega_{i} \mathbb{A}_{i}^{T}: \mathbb{C}_{\text {solid }}: \varepsilon_{i}^{p, n} \\
\boldsymbol{\sigma}_{i}^{n}=\mathbb{C}_{\text {solid }}:\left[\varepsilon_{i}^{n}-\varepsilon_{i}^{p, n}\right] \\
\boldsymbol{\sigma}_{\text {pore }}^{n}=\mathbb{C}_{\text {pore }}: \varepsilon_{\text {pore }}^{n}
\end{gathered}
$$

Changes of stresses and strains during time interval $\Delta t^{n+1}=t^{n+1}-t^{n}$ are quantified in terms of increments

$$
\begin{aligned}
\Delta \mathbf{E}^{n+1} & =\mathbf{E}^{n+1}-\mathbf{E}^{n} \\
\Delta \Sigma^{n+1} & =\Sigma^{n+1}-\boldsymbol{\Sigma}^{n} \\
\Delta \boldsymbol{\sigma}_{i}^{n+1} & =\boldsymbol{\sigma}_{i}^{n+1}-\boldsymbol{\sigma}_{i}^{n} \\
\Delta \varepsilon_{i}^{p, n+1} & =\varepsilon_{i}^{p, n+1}-\varepsilon_{i}^{p, n}
\end{aligned}
$$

The latter increments, relating to microscopic plastic strains, need to be related to the temporal derivatives in the evolution equations (53). Therefore, we employ a backward Euler integration scheme: Except for time derivatives, we hold all functional values fixed at time $t^{n+1}$, and we then integrate the flow rule (53) between time instant $t^{n}$ and $t^{n+1}$, yielding

$$
\begin{aligned}
& \forall i \in \mathcal{S} \\
& \int_{t^{n}}^{t^{n+1}} \dot{\boldsymbol{\varepsilon}}_{i}^{p} d t=\sum_{\alpha=1}^{N_{F a c t, m a x}} \int_{t^{n}}^{t^{n+1}} \dot{\lambda}^{(\alpha)} d t \frac{\partial \mathcal{G}^{(\alpha)}}{\partial \boldsymbol{\sigma}_{i}}\left(\boldsymbol{\sigma}_{i}^{n+1}\right) \\
& =\Delta \varepsilon_{i}^{p, n+1}=\sum_{\alpha=1}^{N_{\text {Fact, max }}} \Delta \lambda_{i}^{(\alpha), n+1} \frac{\partial \mathcal{G}^{(\alpha)}}{\partial \boldsymbol{\sigma}_{i}}\left(\boldsymbol{\sigma}_{i}^{n+1}\right)
\end{aligned}
$$


Table 2: 28 different needle orientations according to integration formulas of Stroud (1971)

\begin{tabular}{|c|c|c|c|c|c|c|c|c|c|c|c|c|c|c|}
\hline$j=$ & 1 & 2 & 3 & 4 & 5 & 6 & 7 & 8 & 9 & 10 & 11 & 12 & 13 & 14 \\
\hline $\sin \left(\theta_{j}\right) \cos \left(\phi_{j}\right)=$ & $+t$ & $+t$ & $-t$ & $-t$ & $+s$ & $+s$ & $-s$ & $-s$ & $+r$ & $+r$ & $-r$ & $-r$ & $+r$ & $+r$ \\
\hline $\sin \left(\theta_{j}\right) \sin \left(\phi_{j}\right)=$ & $+t$ & $-t$ & $+t$ & $-t$ & $+r$ & $-r$ & $+r$ & $-r$ & $+s$ & $-s$ & $+s$ & $-s$ & $+r$ & $-r$ \\
\hline $\cos \left(\theta_{j}\right)=$ & $+t$ & $+t$ & $+t$ & $+t$ & $+r$ & $+r$ & $+r$ & $+r$ & $+r$ & $+r$ & $+r$ & $+r$ & $+s$ & $+s$ \\
\hline$\omega\left(\theta_{j}, \phi_{j}\right)=$ & $a$ & $a$ & $a$ & $a$ & $b$ & $b$ & $b$ & $b$ & $b$ & $b$ & $b$ & $b$ & $b$ & $b$ \\
\hline$j=$ & 15 & 16 & 17 & 18 & 19 & 20 & 21 & 22 & 23 & 24 & 25 & 26 & 27 & 28 \\
\hline $\sin \left(\theta_{j}\right) \cos \left(\phi_{j}\right)=$ & $-u$ & $+v$ & $+v$ & $-v$ & $-v$ & $+v$ & $+v$ & $-v$ & $-v$ & $-r$ & $-r$ & $+u$ & $+u$ & $-u$ \\
\hline $\sin \left(\theta_{j}\right) \sin \left(\phi_{j}\right)=$ & $-v$ & $+u$ & $-u$ & $+u$ & $-u$ & $+v$ & $-v$ & $+v$ & $-v$ & $+r$ & $-r$ & $+v$ & $-v$ & $+v$ \\
\hline $\cos \left(\theta_{j}\right)=$ & $+v$ & $+v$ & $+v$ & $+v$ & $+v$ & $+u$ & $+u$ & $+u$ & $+u$ & $+s$ & $+s$ & $+v$ & $+v$ & $+v$ \\
\hline$\omega\left(\theta_{j}, \phi_{j}\right)=$ & $c$ & $c$ & $c$ & $c$ & $c$ & $c$ & $c$ & $c$ & $c$ & $b$ & $b$ & $c$ & $c$ & $c$ \\
\hline & $\begin{array}{l}\text { with } \\
\text { and }\end{array}$ & $\begin{array}{l}r= \\
u= \\
a=\end{array}$ & 6 & $b=$ & & 8 & $s=$ & & - & & & & $\overline{1 / 3}$ & \\
\hline
\end{tabular}

with the corresponding Melan-Kuhn-Tucker conditions reading as

$$
\begin{array}{ll} 
& \Delta \lambda_{i}^{(\alpha), n+1} \geq 0 \\
& \mathcal{F}^{(\alpha)}\left(\boldsymbol{\sigma}_{i}^{n+1}\right) \leq 0 \\
& \Delta \lambda_{i}^{(\alpha), n+1} \times\left\{1,2, \ldots, N_{\text {Fact }, \text { max }}\right\} \mathcal{F}^{(\alpha)}\left(\boldsymbol{\sigma}_{i}^{n+1}\right)=0
\end{array}
$$

\subsection{Iterative solution process I: macro-to-micro scale transitions}

Inspired by the standard procedure in computational elastoplasticity (Simo and Taylor, 1985; Simo and Hughes, 1998; Hellmich et al., 1999; Morin et al., 2011a,b), a macroscopic strain history is presented in terms of finite strain increments $\Delta \mathbf{E}^{n+1}$, with corresponding totally attained strains reading as

$$
\mathbf{E}^{n+1}=\mathbf{E}^{n}+\Delta \mathbf{E}^{n+1}
$$

These strains can be prescribed directly (displacement-driven situation), or be the result of equilibrium considerations at the RVE or structural level (force-driven situation). Given the full set of state variables at the end of the $n$-th load step, $\Sigma^{n}, \mathbf{E}^{n}, \mathbf{E}^{p, n}$; we are left with computing the same full set of variables for the end of the $(n+1)$-st step, based on the prescribed strain increment $\Delta \mathbf{E}^{n+1}$. Therefore, these macroscopic strains are first downscaled from the macro to the microlevel, under the assumption of the absence of any additional plastic events ("trial state"), on the basis of (59) and (62)

$$
\boldsymbol{\sigma}_{i}^{n+1, \text { trial }}=\mathbb{C}_{\text {solid }}:\left\{\mathbb{A}_{i}: \mathbf{E}^{n+1}+\sum_{j \in \mathcal{S}}\left[\mathbb{D}_{i j}-\delta_{i j} \mathbb{I}\right]: \varepsilon_{j}^{p, n}\right\}
$$


Thereafter, it is checked whether this assumption of purely elastic behavior of all solid phases is actually valid, by inserting the trial state of the microscopic solid microstresses, $\boldsymbol{\sigma}_{i}^{n+1, \text { trial }}$, into the yield criterion $(69)_{2}$, yielding respective trial values

$$
\forall i \in \mathcal{S}, \forall \alpha \in\left\{1,2, \ldots, N_{\text {Fact }, \text { max }}\right\} \quad \mathcal{F}_{i}^{(\alpha), n+1, \text { trial }}=\mathcal{F}^{(\alpha)}\left(\boldsymbol{\sigma}_{i}^{n+1, \text { trial }}\right)
$$

with $N_{\text {Fact,max }}$ as the maximum number of potentially active yield surfaces per solid phase. These values allow for discrimination between plasticizing and purely elastic phases; as well as between active and non-active yield surfaces associated to these plasticizing phases: all solid phases with $\mathcal{F}_{i}^{(\alpha), n+1, \text { trial }} \leq 0$ holding for all $N_{\text {Fact,max }}$ yield surfaces associated to these solid phases, behave purely elastically during time step $\Delta t^{n+1}$. On the other hand, those with $\mathcal{F}_{i}^{(\alpha), n+1, \text { trial }}>0$, for at least one of the $N_{\text {Fact,max }}$ yield surfaces defining the multisurface criterion of each solid phase, define the set of plasticizing needle-shaped solid phases, $\mathcal{S}_{\text {cyl,plast }}$. This set has $N_{c y l, p l a s t}$ elements, $i=1, . ., N_{c y l, p l a s t}$. Each element of this set is associated to a number of active yield surfaces, $N_{\text {Fact }, i}$; always fulfilling $\mathcal{F}_{i}^{(\alpha), n+1, \text { trial }}>0$. These plasticizing phases exhibit non-zero plastic strain increments $\Delta \varepsilon_{i}^{p, n+1}$ according to flow rule (68), which are determined from fulfillment of the Melan-KuhnTucker conditions (69), while considering state equation (62) evaluated at load step $(n+1)$ and the definition of the trial state according to (71),

$\forall i \in \mathcal{S}_{\text {cyl }, \text { plast }}, \forall \alpha \in\left\{1,2, \ldots, N_{\text {Fact }, i}\right\}$

$\mathcal{F}^{(\alpha)}\left(\boldsymbol{\sigma}_{i}^{n+1, \text { trial }}+\mathbb{C}_{\text {solid }}:\left\{\sum_{j \in \mathcal{S}_{\text {cyl }, \text { plast }}} \sum_{\beta=1}^{N_{\text {Fact }, j}}\left[\mathbb{D}_{i j}-\delta_{i j} \mathbb{I}\right] \Delta \lambda_{j}^{(\beta), n+1} \frac{\partial \mathcal{G}^{(\beta)}}{\partial \boldsymbol{\sigma}_{j}}\left(\boldsymbol{\sigma}_{j}^{n+1}\right)\right\}\right)=0$

Due to the nonlinear dependence of the phase-specific plastic flow directions $\frac{\partial \mathcal{G}^{(\beta)}}{\partial \boldsymbol{\sigma}_{j}}$ on the phase stresses $\boldsymbol{\sigma}_{j}$, a solution for $\Delta \lambda_{j}^{(\beta), n+1}$ cannot be directly gained from (73). Therefore, we solve (73) in an iterative manner, with iteration steps labelled by $k$. Thereby, the approximations of the first iteration step $(k=1)$ relate to the trial state and therefore read as

$$
\begin{aligned}
& \Delta \boldsymbol{\varepsilon}_{i}^{p, n+1,1}=0 \\
& \Delta \lambda_{i}^{(\alpha), n+1,1}=0 \\
& \boldsymbol{\sigma}_{i}^{n+1,1}=\boldsymbol{\sigma}_{i}^{n+1, \text { trial }}
\end{aligned}
$$


They are used for approximating the plastic flow direction, as the basis for obtaining an improved estimate for the plastic multiplier $\Delta \lambda_{j}^{(\alpha), n+1, k+1}$, according to the approximation scheme

$$
\begin{aligned}
& \forall i \in \mathcal{S}_{\text {cyl }, \text { plast }}, \forall \alpha \in\left\{1,2, \ldots, N_{\text {Fact }, i}\right\} \\
& \mathcal{F}^{(\alpha)}\left(\boldsymbol{\sigma}_{i}^{n+1, \text { trial }}\right. \\
& \left.+\mathbb{C}_{\text {solid }}:\left\{\sum_{j \in \mathcal{S}_{\text {cyl }, \text { plast }}} \sum_{\beta=1}^{N_{\text {Fact }, j}}\left[\mathbb{D}_{i j}-\delta_{i j} \mathbb{I}\right] \Delta \lambda_{j}^{(\beta), n+1, k+1} \frac{\partial \mathcal{G}^{(\beta)}}{\partial \boldsymbol{\sigma}_{j}}\left(\boldsymbol{\sigma}_{j}^{n+1, k}\right)\right\}\right)=0
\end{aligned}
$$

Solution of this non-linear equation (77) requires yet another iteration scheme (described in the next section), providing new (improved) approximative solutions $\Delta \lambda_{i}^{(\beta), n+1, k+1}, i=1, \ldots, N_{\text {cyl }, \text { plast }}, \beta=1, \ldots, N_{F a c t, i}$ with corresponding (improved) plastic strains

$$
\begin{aligned}
& \forall i \in\left\{1, \ldots, N_{\text {cyl }, \text { plast }}\right\} \\
& \Delta \varepsilon_{i}^{p, n+1, k+1}=\sum_{\alpha=1}^{N_{\text {Fact }, i}} \Delta \lambda_{i}^{(\alpha), n+1, k+1} \frac{\partial \mathcal{G}^{(\alpha)}}{\partial \boldsymbol{\sigma}_{i}}\left(\boldsymbol{\sigma}_{i}^{n+1, k}\right)
\end{aligned}
$$

Update of the corresponding microscopic stresses requires another downscaling process from the macro to the microlevel, according to

$$
\begin{aligned}
& \forall i \in\left\{1, \ldots, N_{\text {cyl }, \text { plast }}\right\} \\
& \boldsymbol{\sigma}_{i}^{n+1, k+1}=\boldsymbol{\sigma}_{i}^{n+1, \text { trial }} \\
& +\mathbb{C}_{\text {solid }}:\left\{\sum_{j \in \mathcal{S}_{\text {cyl }, \text { plast }}} \sum_{\alpha=1}^{N_{F a c t, j}}\left[\mathbb{D}_{i j}-\delta_{i j} \mathbb{I}\right] \Delta \lambda_{j}^{(\alpha), n+1, k+1} \frac{\partial \mathcal{G}^{(\alpha)}}{\partial \boldsymbol{\sigma}_{j}}\left(\boldsymbol{\sigma}_{j}^{n+1, k}\right)\right\}
\end{aligned}
$$

Thereafter, iteration step $(k+1)$ is completed by checking whether the alterations in the plastic flow stemming from the update of $\boldsymbol{\sigma}_{i}^{n+1}$ from iteration step $k$ to iteration step $(k+1)$ become negligible, i.e. lower than a prescribed tolerance value TOL,

$$
\begin{aligned}
& \forall i \in\left\{1, \ldots, N_{\text {cyl plast }}\right\}, \forall \alpha \in\left\{1,2, \ldots, N_{\text {Fact }, i}\right\} \\
& \left\|\frac{\partial \mathcal{G}^{(\alpha)}}{\partial \boldsymbol{\sigma}_{i}}\left(\boldsymbol{\sigma}_{i}^{n+1, k+1}\right)-\frac{\partial \mathcal{G}^{(\alpha)}}{\partial \boldsymbol{\sigma}_{i}}\left(\boldsymbol{\sigma}_{i}^{n+1, k}\right)\right\| \leq \mathrm{TOL}
\end{aligned}
$$


If this is not the case yet, the $(k+1)$-st approximations are used as input for the next (i.e. $(k+2)$-nd) iteration, which is characterized by replacement of $k$ by $(k+1)$, and of $(k+1)$ by $(k+2)$, in $(77),(78),(79)$ and $(80)$.

\subsection{Iterative process II: plastic flow - "return map"}

In each iteration step $k$ described in the preceding section, the non-linear equation

$$
\begin{aligned}
& \forall i \in \mathcal{S}_{\text {cyl }, \text { plast }}, \forall \alpha \in\left\{1,2, \ldots, N_{\text {Fact }, i}\right\} \\
& \mathcal{F}^{(\alpha)}\left(\tilde{\boldsymbol{\sigma}}_{i}^{n+1}=\boldsymbol{\sigma}_{i}^{n+1, \text { trial }}+\right. \\
& \left.+\mathbb{C}_{\text {solid }}:\left\{\sum_{j \in \mathcal{S}_{\text {cyl }, \text { plast }}} \sum_{\beta=1}^{N_{\text {Fact }, j}}\left[\mathbb{D}_{i j}-\delta_{i j} \mathbb{I}\right] \Delta \lambda_{j}^{(\beta), n+1, k+1} \frac{\partial \mathcal{G}^{(\beta)}}{\partial \boldsymbol{\sigma}_{j}}\left(\boldsymbol{\sigma}_{j}^{n+1, k}\right)\right\}\right)=0
\end{aligned}
$$

needs to be solved. This is done by a Newton iteration scheme, with iteration steps labelled by $l$, reading as

$$
\mathbf{R}^{n+1, k+1, l}+\frac{d \mathbf{R}^{n+1, k+1, l}}{d\left(\Delta \boldsymbol{\lambda}^{n+1, k+1}\right)} \cdot \Delta(\Delta \boldsymbol{\lambda})^{n+1, k+1, l+1}=0
$$

Thereby, the vectorial terms in (82) are defined as follows: The plastic multiplier vector reads as

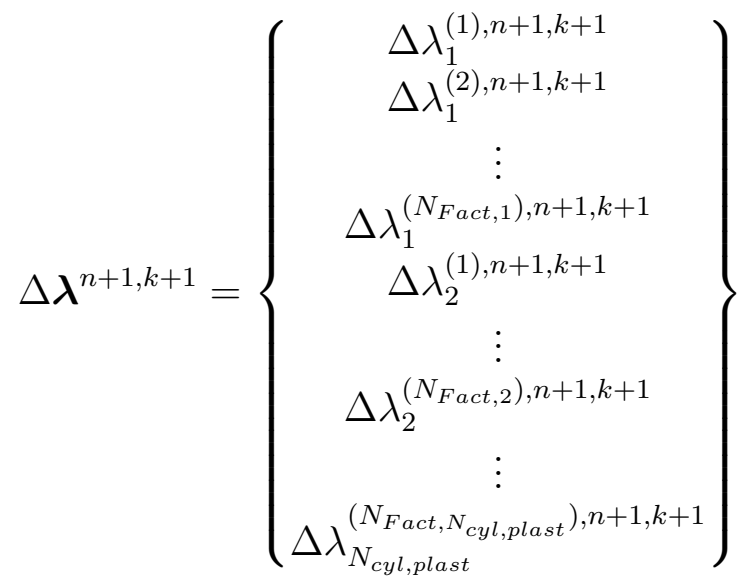


The residual vector reads as

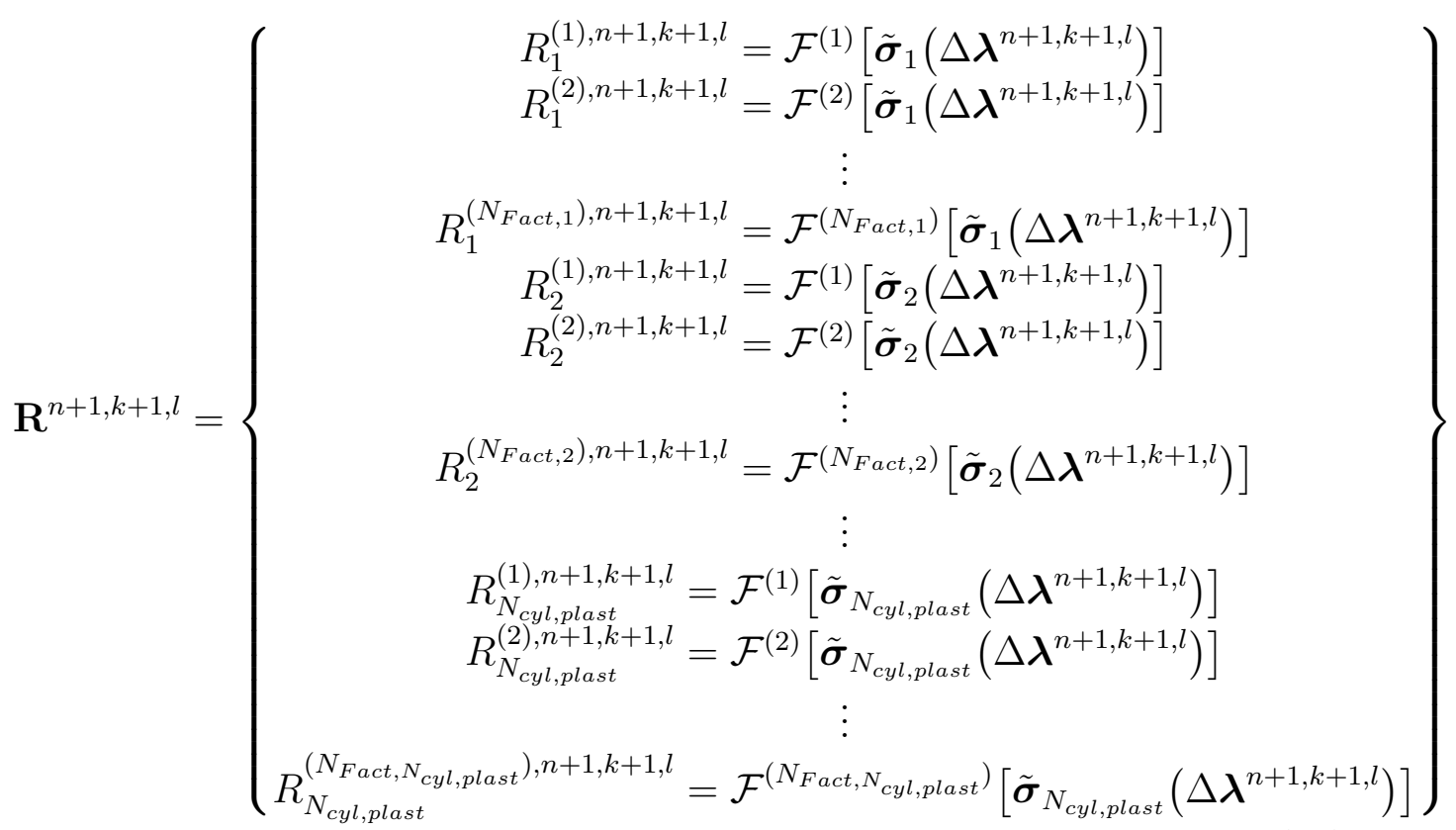

with $N_{\text {Fact }, i}$ as the number of active surfaces of phase $i$; and its derivatives

$$
\begin{aligned}
& \frac{d \mathbf{R}^{n+1, k+1, l}}{d\left(\boldsymbol{\lambda}^{n+1, k+1}\right)}=
\end{aligned}
$$

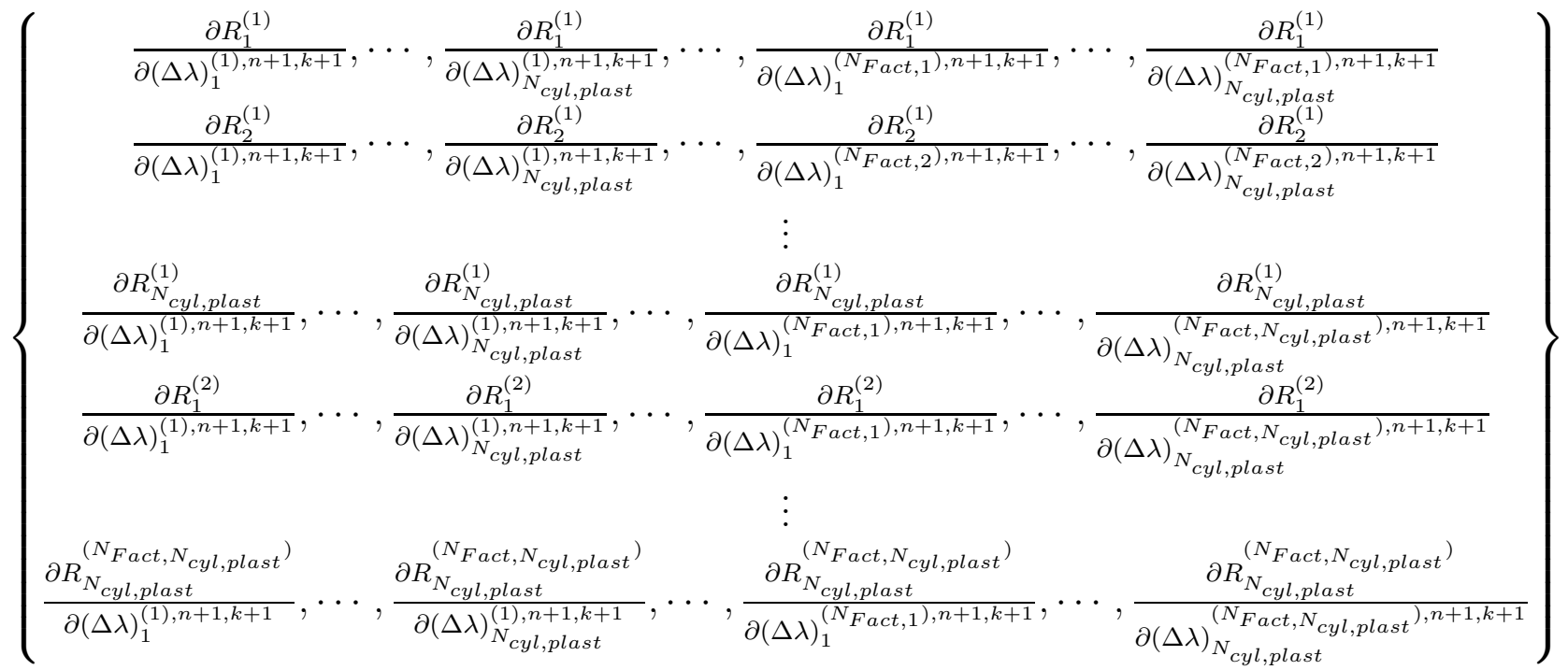


whereby

$$
\begin{aligned}
\frac{\partial R_{1}^{(\beta)}}{\partial\left(\Delta \lambda_{i}^{(\alpha), n+1, k+1}\right)} & =\frac{\partial \mathcal{F}^{(\beta)}}{\partial \tilde{\boldsymbol{\sigma}}_{1}^{n+1}}\left(\tilde{\boldsymbol{\sigma}}_{1}^{n+1}\right): \frac{\partial \tilde{\boldsymbol{\sigma}}_{1}}{\partial(\Delta \lambda)_{i}^{(\alpha), n+1, k+1}} \\
& =\frac{\partial \mathcal{F}^{(\beta)}}{\partial \tilde{\boldsymbol{\sigma}}_{1}^{n+1}}\left(\tilde{\boldsymbol{\sigma}}_{1}^{n+1}\right): \mathbb{C}_{\text {solid }}:\left[\mathbb{D}_{1 i}-\delta_{1 i} \mathbb{I}\right]: \frac{\partial \mathcal{G}^{(\alpha)}}{\partial \tilde{\boldsymbol{\sigma}}_{i}}\left(\boldsymbol{\sigma}_{i}^{n+1, k}\right)
\end{aligned}
$$

The solution vector of iteration $(l+1)$ reads as

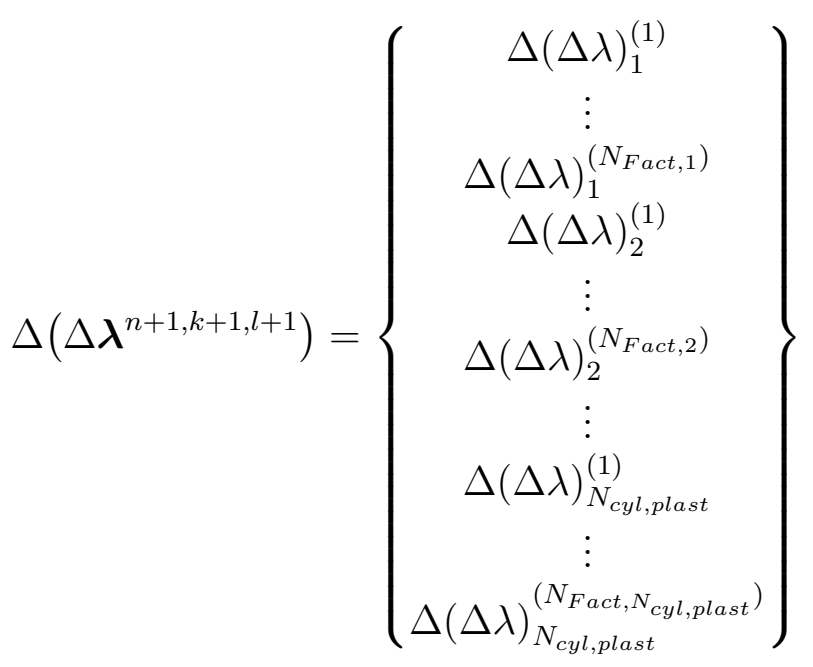

and the updated plastic multiplier vector as

$$
\Delta \boldsymbol{\lambda}^{n+1, k+1, l+1}=\Delta \boldsymbol{\lambda}^{n+1, k+1, l}+\Delta(\Delta \boldsymbol{\lambda})^{n+1, k+1, l+1}
$$

If the converged solutions $\Delta(\Delta \lambda)_{i}^{(\alpha), n+1}$ are all positive, the original guess of active phases and surfaces according to the trial state definition (71) and (72) was correct. If any $\Delta(\Delta \lambda)_{i}^{(\alpha), n+1}$ are negative, the corresponding yield surfaces are discarded from the sets $\mathcal{S}_{\text {cyl,plast }}$ and $\left\{1,2, \ldots, N_{\text {Fact }, i}\right\}$ and the process (73) to (88) is repeated as long as all plastic multipliers are nonnegative.

\section{Exemplification of multiscale elastoplasticity through upscaling of Mohr-Coulomb criterion}

We here exemplify the developments of Section 5 by assigning, to all needle-shaped solid phases of Figure 1, a Mohr-Coulomb criterion in the form

$$
\mathcal{F}(\boldsymbol{\sigma})=\tau-\left(c_{\text {solid }}-\sigma \tan \varphi_{\text {solid }}\right) \leq 0
$$


with $\tau=\sigma_{n t}$ and $\sigma=\sigma_{n n}$ as the components of the traction vector $\mathbf{T}=\boldsymbol{\sigma} \cdot \mathbf{n}$ in the so-called Mohr plane, which is spanned by the vectors $\mathbf{n}$ and $\mathbf{t}$. $\mathbf{t}$ is in this Mohr plane as well, arising from rotating $\mathbf{n}$ by $(\pi / 2)$; i.e. orthogonal to n. Upon fulfillment of (89), plastic strains may occur only in terms of shear components $\varepsilon_{n t}^{p}$, i.e. only such strain components which are energetically conjugated to $\tau=\sigma_{n t}$. According to the general flow rule of (53), this may be expressed by a plastic potential $\mathcal{G}$ which does not depend on the normal stress component $\sigma$, hence being of the form

$$
\mathcal{G}(\boldsymbol{\sigma})=\tau
$$

so that $\partial \mathcal{G} / \partial \sigma=0$ and $\partial \mathcal{G} / \partial \tau=1$. In the principle stress state, (89) represents a pyramid with six faces, six edges and an apex; while (90) represents a prism with six faces and edges, being oriented parallel to the hydrostatic axis. Back-projection of trial stress states on these edges and faces of the pyramid, along directions orthogonal to the edges and faces of the prism can be conveniently performed on the basis of the following multisurface representation of (89) and (90) in the principal stress space; comprising three yield functions

$$
\begin{aligned}
& \mathcal{F}^{(1)}=\beta \sigma_{i, I}-\sigma_{i, I I I}-\sigma_{y} \\
& \mathcal{F}^{(2)}=\beta \sigma_{i, I}-\sigma_{i, I I}-\sigma_{y} \\
& \mathcal{F}^{(3)}=\beta \sigma_{i, I I}-\sigma_{i, I I I}-\sigma_{y}
\end{aligned}
$$

with the friction parameter $\beta$ and the compressive yield stress $\sigma_{y}$ being related to the cohesion $c_{\text {solid }}$ and the angle of internal friction $\varphi_{\text {solid }}$ through

$$
\sigma_{y}=\frac{2 c_{\text {solid }} \cos \varphi_{\text {solid }}}{1-\sin \varphi_{\text {solid }}}
$$

and

$$
\beta=\frac{1+\sin \varphi_{\text {solid }}}{1-\sin \varphi_{\text {solid }}}
$$

and the plastic potential functions

$$
\begin{aligned}
& \mathcal{G}^{(1)}=\sigma_{i, I}-\sigma_{i, I I I} \\
& \mathcal{G}^{(2)}=\sigma_{i, I}-\sigma_{i, I I} \\
& \mathcal{G}^{(3)}=\sigma_{i, I I}-\sigma_{i, I I I}
\end{aligned}
$$


whereby $\sigma_{i, I} \geq \sigma_{i, I I} \geq \sigma_{i, I I I}$ are the principal stresses of stress tensor $\boldsymbol{\sigma}_{i}$. (91) and (94) can be readily inserted into the general form of the algorithm (70)-(88).

We explicitly note that the yield and potential functions (91) and (94) appear as linear in the principal stress state, but that the corresponding principal directions do change during the loading and back-projection steps, which renders the problem as fully non-linear. Depending on the degree of nonlinearity, the accuracy of the trial state in correctly targeting the actually plastically active solid phases and yield surfaces may reduce, which then prolongs the computation time. In this context, the introduction of plastic stress regions according to Clausen et al. (2007) turns out as helpful: These regions are defined on the basis of two expressions, which relate to the delimiting lines of semi-infinite triangular plane (to which the Mohr-Coulomb criterion degenerates in the principical stress space, once the principal stresses are ordered according to $\left.\sigma_{I} \geq \sigma_{I I} \geq \sigma_{I I I}\right)$. They are

$$
\begin{aligned}
p_{I-I I}(\boldsymbol{\sigma}) & =\frac{1}{\beta+1}\left(\sigma_{I}-\frac{\sigma_{y}}{\beta-1}\right)-\left(\sigma_{I I}-\frac{\sigma_{y}}{\beta-1}\right) \\
& +\frac{1}{\beta+1}\left(\sigma_{I I I}-\frac{\sigma_{y}}{\beta-1}\right) \\
p_{I-I I I}(\boldsymbol{\sigma}) & =\frac{\beta}{\beta+1}\left(\sigma_{I}-\frac{\sigma_{y}}{\beta-1}\right)-\left(\sigma_{I I}-\frac{\sigma_{y}}{\beta-1}\right) \\
& +\frac{\beta}{\beta+1}\left(\sigma_{I I I}-\frac{\sigma_{y}}{\beta-1}\right)
\end{aligned}
$$

The aforementioned stress regions for categorization of the trial stress states are:

- Stress region I relates to $p_{I-I I}\left(\boldsymbol{\sigma}_{i}^{n+1, \text { trial }}\right) \geq 0$ and $p_{I-I I I}\left(\boldsymbol{\sigma}_{i}^{n+1, \text { trial }}\right) \leq$ 0 : if the trial stress resides in this region, then we choose $\mathcal{F}^{(1)}$ according to (91) as active yield surface.

- Stress region II relates to $p_{I-I I}\left(\boldsymbol{\sigma}_{i}^{n+1, \text { trial }}\right)<0$ and $p_{I-I I I}\left(\boldsymbol{\sigma}_{i}^{n+1, \text { trial }}\right)<$ 0: if the trial stress resides in this region, then we choose $\mathcal{F}^{(1)}$ and $\mathcal{F}^{(2)}$ according to (91) as active yield surfaces.

- Stress region III relates to $p_{I-I I}\left(\boldsymbol{\sigma}_{i}^{n+1, \text { trial }}\right)>0$ and $p_{I-I I I}\left(\boldsymbol{\sigma}_{i}^{n+1, \text { trial }}\right)>$ 
0: if the trial stress resides in this region, then we choose $\mathcal{F}^{(1)}$ and $\mathcal{F}^{(3)}$ according to (91) as active yield surfaces.

- Stress region IV relates to all other cases, where we choose all three yield surfaces as active.

Finally, the actually active yield surfaces are idenfitied as described at the end of Section 5.3.

Next, we show the application of the algorithm described in Section 5 and applied to Mohr-Coulomb plasticity earlier in the present chapter, for computing the behavior of the RVE shown in Figure 1 WITH MATERIAL PROPERTIES COLLECTED IN TABLE 3 , under pure shear, $\boldsymbol{\Sigma}=\Sigma_{23}\left(\mathbf{e}_{2} \otimes \mathbf{e}_{3}+\mathbf{e}_{3} \otimes \mathbf{e}_{2}\right)$, under uniaxial tension, $\boldsymbol{\Sigma}=\Sigma_{33} \mathbf{e}_{3} \otimes \mathbf{e}_{3}, \Sigma_{33}>0$, and under uniaxial compression, $\boldsymbol{\Sigma}=\Sigma_{33} \mathbf{e}_{3} \otimes \mathbf{e}_{3}, \Sigma_{33}<0$.

Table 3: Properties of SOlid AND PORE MATERIAl Phases, FOR THE CASE OF POROUS HYDROXYAPATITE POLYCRYSTALS, SEE SECTION 7 FOR EXPERIMENTAL DETAILS

\begin{tabular}{l|cc} 
Property & Solid phases & Pore phases \\
\hline Bulk modulus & $k_{\text {solid }}=82.6 \mathrm{GPa}$ & $k_{\text {pore }}=2.3 \mathrm{GPa}$ \\
Shear modulus & $\mu_{\text {solid }}=44.9 \mathrm{GPa}$ & $\mu_{\text {pore }}=0 \mathrm{GPa}$ \\
Cohesion & $c_{\text {solid }}=82.2 \mathrm{MPa}$ & \\
Friction angle & $\varphi=57.8^{\circ}$ & \\
Volume fraction & $f_{H A}=0.68$ & $f_{\text {pore }}=0.32$
\end{tabular}

First of ALL, IT IS INTERESTING TO STUDY THE EFFECT OF DIFFERENT DISCRETIZATION SCHEMES ON THE MODEL PREDICTIONS, SEE FIGURES 4(A), 5(A), AND 6(A). TWO REALizATIONS OF THE INTEGRATION SCHEME OF STROUd (1971), WITH 15 AND 28 INTEGRATION POINTS, RESPECTIVELY, AS WELL AS THE METHOD OF BADEL AND LEBLOND (2004) WITH 120 INTEGRATION POINTS, DELIVER VERY SIMILAR RESULTS - INDICATING THE CONVERGED NATURE OF THE REPORTED COMPUTATIONAL RESUlts (SEE TABLE 4). For all investigated load cases, plasticity leads to non-linear macroscopic stress-strain curves, and this non-linearity is associated with the propagation of plasticity throughout the solid needle phases. AS ALL SOLID NEEDLE PHASES EXHIBIT IDEAL PLASTIC BEHAVIOR, THEY CANNOT UNDERGO ANY UNLOADING PROCESSES UNDER MACROSCOPIC 


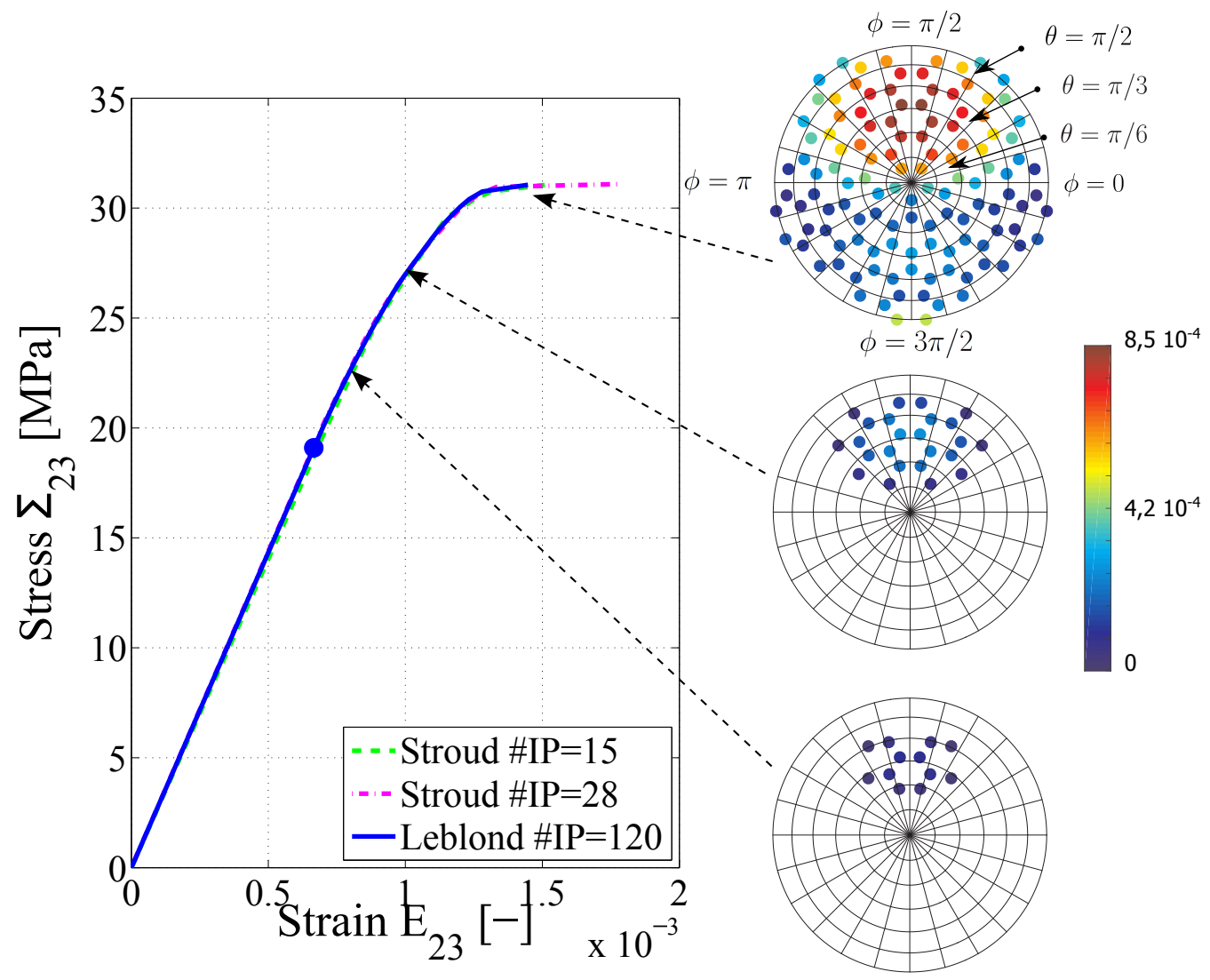

Figure 4: (a) Macroscopic stress-strain relation under pure shear (depicted from the onset of plastic events), for different discretizations according to Stroud (1971) and Badel and Leblond (2004), IP... integration point; (b) spreading of plasticity throughout solid needle-shaped phases, as function of macroscopic shear stress; (c) evolution of micro-plastic region in the solid phase orientation space, as function of the macroscopic pure shear stress as indicated in the color code

LOADING STATES. With increasing macroscopic stress, an increasing fraction of the solid needle phases plasticize, as illustrated in Figures 4(b), 5(b), and $6(\mathrm{~b})$. The orientation of these increasing number of plasticizing phases, quantified in terms of the angles $\theta$ and $\phi$ given in Figure 2, can be illustrated on the stereographic projection of a unit sphere onto a plane parallel 


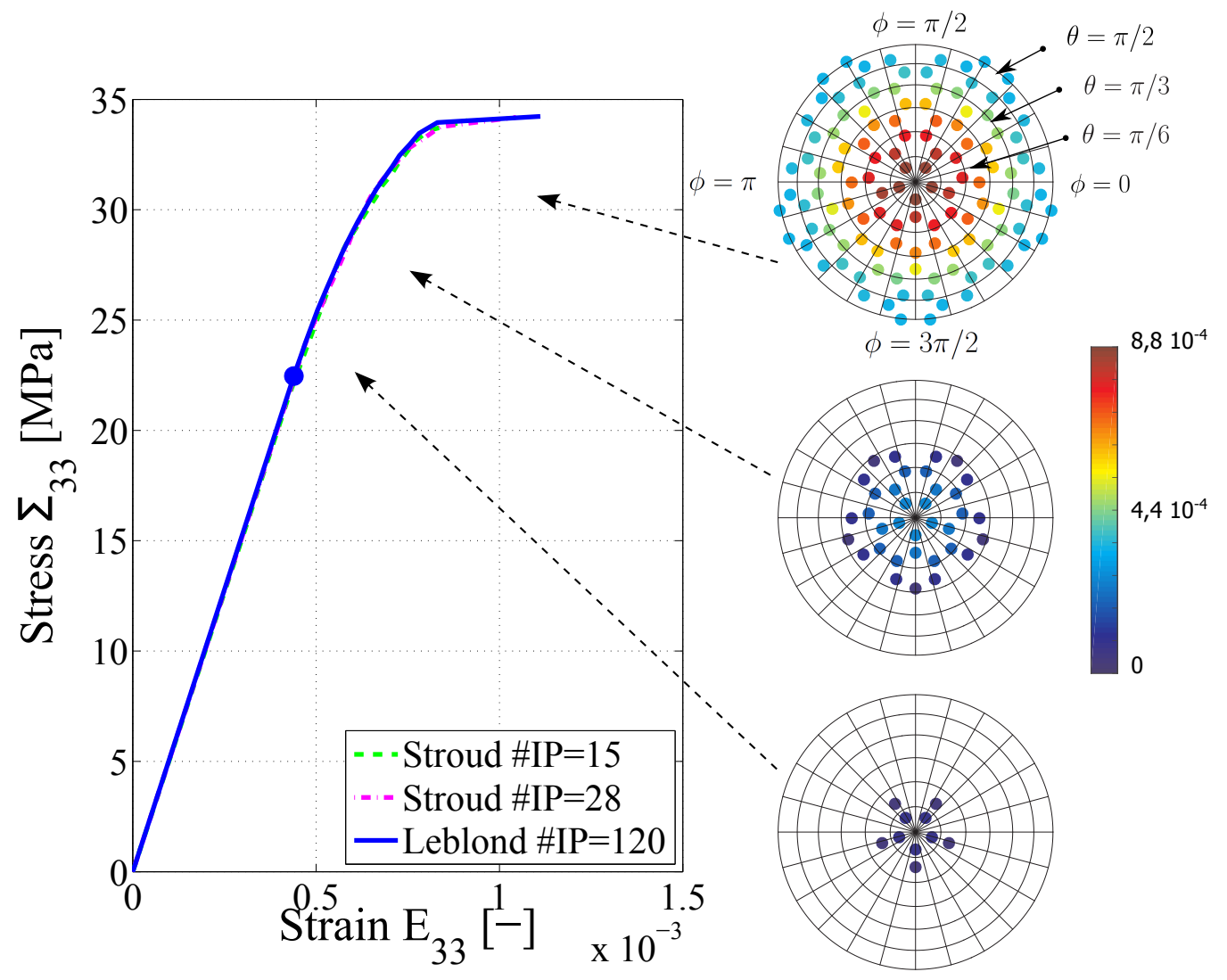

Figure 5: (a) Macroscopic stress-strain relation under uniaxial tension (depicted from the onset of plastic events), for different discretizations according to Stroud (1971) and Badel and Leblond (2004), IP... integration point; (b) spreading of plasticity throughout solid needle-shaped phases, as function of macroscopic uniaxial tensile stress; (c) evolution of microplastic region in the solid phase orientation space, as function of the macroscopic uniaxial tensile stress as indicated in the color code

to the base vectors $\mathbf{e}_{1}$ and $\mathbf{e}_{2}$, see Figures 4(c), 5(c), and 6(c). In such a circular projection, different values for the co-latitudinal coordinate $\theta$ are associated with different concentric circles, the outermost circle being related to $\theta=\pi / 2$; and different values for the longitudinal coordinate $\phi$ are associ- 


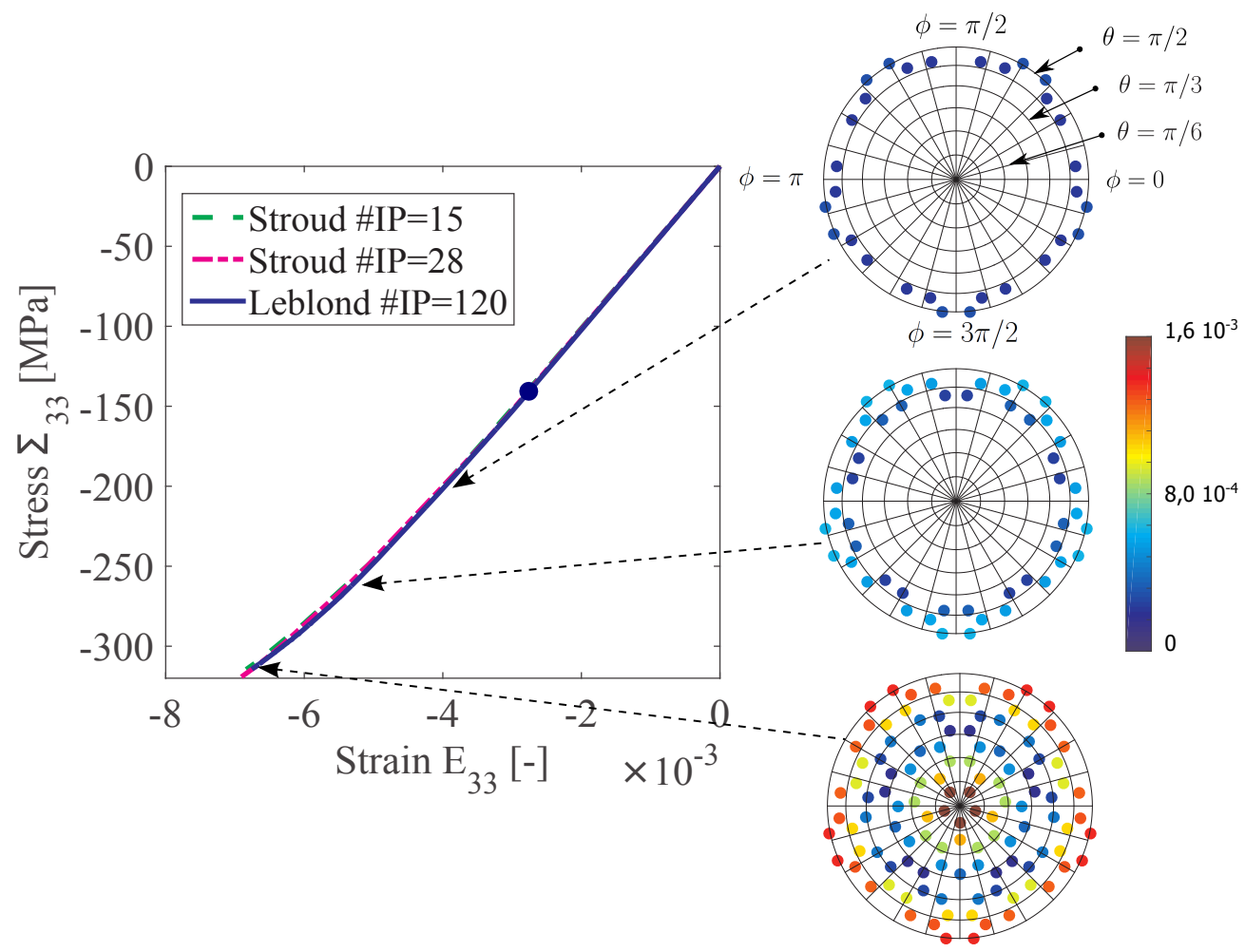

Figure 6: (a) Macroscopic stress-strain relation under uniaxial compression (depicted from the onset of plastic events), for different discretizations according to Stroud (1971) and Badel and Leblond (2004), IP... integration point; (b) spreading of plasticity throughout solid needle-shaped phases, as function of macroscopic uniaxial compressive stress; (c) evolution of micro-plastic regions in the solid phase orientation space, as function of the macroscopic uniaxial compressive stress as indicated in the color code

ated with different straight lines oriented orthogonal to the aforementioned circles. Each macroscopic stress level indicated on the ordinates of the diagrams in Figures 4(b), 5(b), and 6(b) is associated with plastic regions on the aforementioned projection area, seen in Figures 4(c), 5(c), and 6(c). These plastic regions are indicated through the color code seen in Figures 4(c), 5(c), and 6(c): at the onset of plastic events, these regions are indicated in red, and 
Table 4: Yield AND Ultimate MACROSCOPIC STRESSES [IN MPA] COMPUTED FOR UNIAXIAL TENSION, UNIAXIAL COMPRESSION, AND PURE SHEAR: BASED ON DIFFERENT DISCRETIZATION SCHEMES AND DIFFERENT NUMBERS OF INTEGRATION POINTS (\#IP)

\begin{tabular}{l|cccc} 
Discretization & Load case & & Yield stress & Ultimate stress \\
\hline \multirow{3}{*}{ Stroud \#IP=15 } & uniaxial tension & $\Sigma_{33}=$ & 21.61 & 34.04 \\
& uniaxial compression & $\Sigma_{33}=$ & -131.50 & -314.50 \\
& pure shear & $\Sigma_{23}=$ & 20.87 & 31.05 \\
\hline \multirow{3}{*}{ Stroud \#IP=28 } & uniaxial tension & $\Sigma_{33}=$ & 23.77 & 34.20 \\
& uniaxial compression & $\Sigma_{33}=$ & -151.77 & -319.16 \\
& pure shear & $\Sigma_{23}=$ & 20.01 & 31.10 \\
\hline \multirow{3}{*}{ Leblond \#IP=120 } & uniaxial tension & $\Sigma_{33}=$ & 22.41 & 34.26 \\
& uniaxial compression & $\Sigma_{33}=$ & -139.84 & -313.84 \\
& pure shear & $\Sigma_{23}=$ & 19.09 & 31.06
\end{tabular}

with increasing macroscopic stress level, they are indicated by more and more different colors: first red and orange; then red, orange, and yellow; thereafter red, orange, yellow, and green; and finally red, orange, yellow, green, and blue, see Figures 4(c), 5(c), and 6(c). Thereby, these plastic regions obviously represent the orientations of all the solid needle-shaped phases which are undergoing plastic events. Once the blue color scale is reached, all solid phases plasticize, and the RVE would fail under a macroscopic stress-driven test. Naturally, the evolution of the aforementioned plastic regions in Figures $4(\mathrm{c}), 5(\mathrm{c})$, and $6(\mathrm{c})$ give interesting insights into the microstructural events arising under macroscopic loading of the types pure shear, uniaxial tension, and uniaxial compression: In pure shear (see Figure 4(c)), plasticity starts in needles belonging to the shear plane and inclined by $\pi / 4$ to the $\mathbf{e}_{2}$ - $\mathbf{e}_{3}$-axes, respectively, and propagates towards needles oriented off-plane, but also less inclined from the axes $\mathbf{e}_{2}$ and $\mathbf{e}_{3}$. Under macroscopic uniaxial tensile loading in $\mathbf{e}_{3}$-direction $(\theta=0)$, the needle-shaped phases oriented in loading directions are the first to plastify, and the subsequent plastification process spreads axisymmetrically around $\mathbf{e}_{3}$, see Figure 5(c). In uniaxial compression (see Figure 6(c)), plasticity starts in needles orthogonal to the load direction, AND WHILE THIS PLASTIC REGION ON THE UNIT SPHERE PROJECTION AREA SPREADS, A SECOND, INDEPENDENT NON-CONTIGUOUS PLASTIC REGION EMERGES AROUND $\theta=0$, I.E. NEEDLES ORIENTED IN THE 
LOADing DiRECTiOn START TO Plasticize as Well. Finally, PlastiFICATION EVENTS AFFECT ALSO THE NEEDLE-SHAPED PHASES INCLINED BY $\pi / 4$ FROM THE LOADING DiRECTION, SEE FiguRE 6(C).

\section{Application: Push-out test on osteonal bone}

Bone tissue is a hierarchically organized material composed of three elementary COMPONENTS GOVERNING ITS MECHANICAL BEHAVIOR: mineral, collagen, and water with non-collageneous organics. These elementary components are arranged in different microstructures, from the nanometer scale up to that of milimeters (Katz et al., 1984; Weiner and Wagner, 1998), see Figure 7. The seemingly "compact", so-called cortical shell surrounding the macroscopic organ [see Figure 7(a)] appears, under greater magnification, as a porous material itself, with cylindrical pores (called Haversian canals) being surrounded by concentric layers of lamellar bone matrix (see Figure 7(b) for the corresponding microstructural unit called "osteon"). Each osteon consists of one Haversian canal surrounded by alternating collagen-rich and collagen-poor layers of extravascular bone matrix (Marotti et al., 1994, 2013), AND SUCH AN OSTEON IS BOUNDED BY A SO-CALLED CEMENT LINE With up to 5 MiCrons thickness (Skedros ET AL., 2005). This CEMENT LINE IS COLLAGEN-FREE, AS WAS EXPERIMENTALLY EVIDENCED BY Staining tests (Weidenreich, 1930; Weinmann And Sicher, 1955; Sokoloff, 1973; Skedros et Al., 2005). Conclusively, Cement LINES CONTAIN HYDROXYAPATITE AND WATER WITH NONCOLLAGENEOUS ORGANICS, THESE COMPONENTS MAKING UP A POLYCRYSTALLINE MATERial as SEEN IN Figure 7(C). This MATERIAL CAN BE SUITABly REPRESEnted By the RVE of Figure 1, SeE Figure 7(D). Such an RVE HAS BEEN EXTENSIVELY USED FOR THE MICROMECHANICAL MODELING OF THE EXTRAFIBRILLAR SPACES WITHIN THE BONE ULTRASTRUCTURE (Fritsch et Al., 2009A; Vuong And Hellmich, 2011; Morin And Hellmich, 2014).

The microscopic strength properties of the osteon can be determined through push-out tests, first carried out by Ascenzi and Bonucci (1972) on bone from human femoral shafts, and later by Bigley et al. (2006) on bone from the third metapcarpal of a racehorse. During such tests, a punch with a diameter of about $150 \mu \mathrm{m}$ applies a compressive load on the osteon until its complete debonding. The ABSEnCE OF COLlaGen IN THE CEMENT LINES MAKES 


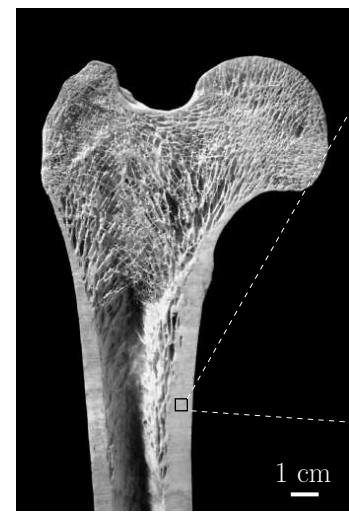

(a)

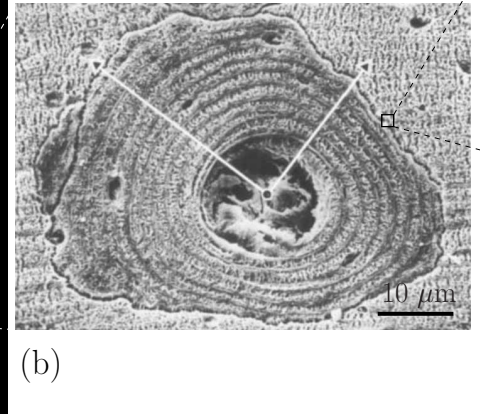

(d)

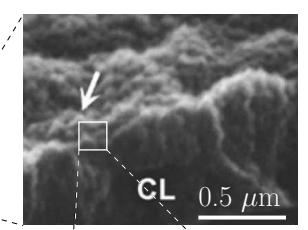

(c)

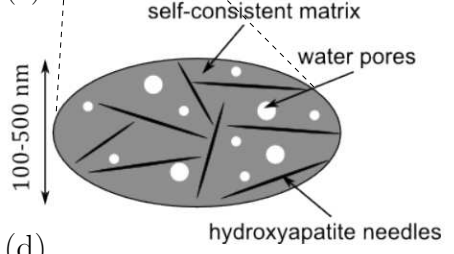

hydroxyapatite needles

Figure 7: Hierarchical organization of bone: (A) PHOTOGRAPH OF A WHOLE BONE; (B) SCANNING ELECTRON MICROGRAPH OF AN OSTEON, BEING COMPOSED OF ALTERNATING THICK (DENSE, COLlAGEN-RICH) AND THIN (LOOSE, COLlAGENPOOR) LAMELLAE, AND DELINEATED BY A COLLAGENFREE CEMENT LINE (MAROTTI, 1993), (C) SCANNING ELECTRON MICROGRAPH OF THE ISOLATED CEMENT LINE (DAVIES, 2007), (D) MICROMECHANICAL REPRESENTATION OF COLLAGEN-FREE COMPARTMENT OF BONE ULTRASTRUCTURE, ALSO REFERRED TO AS "EXTRAFIBRILLAR SPACE" (PROSTAK AND LEES, 1996); PERMission FOR IMAge Reproduction Requested (A) From PAul Crompton, University of Wales College of Medicine, (B) from Springer-Verlag New York Inc. For Figures 1 of (Marotti, 1993), AND (C) From ElSEVIER FOR Figure 2B of (DAvies, 2007)

THEM COMPARATIVELY WEAK, AND RENDERS THEM AS PREFERRED LOCATIONS OF OSTEON DEBONDING IN THE COURSE OF PUSH-OUT TESTS.

Such a push-out test produces an pure shear stress in the cement line, which we prescribe in the form $\boldsymbol{\Sigma}=\Sigma_{23}\left(\mathbf{e}_{2} \otimes \mathbf{e}_{3}+\mathbf{e}_{3} \otimes \mathbf{e}_{2}\right)$ on the RVE of Figure 1 with solid phases following a Mohr-Coulomb criterion; by using the algorithm described in Section 5. As model input parameters, we need (i) the elastic properties of of the needle-shaped phases and of the pores, (ii) the strength properties of the plastic solid phases, and (iii) the volume fractions 
of pores (FILLED BY WATER AND NON-COLLAGENEOUS ORGANIC MATTER) and solid phases (made of hydroxyapatite, abbreviated as HA).

The elastic properties of the constituents were determined by experiments performed on pure constituents: ultrasonic measurements on hydroxyapatite allowed to identify the following isotropic properties (Katz and Ukraincik, 1971; Gilmore and Katz, 1982)

$$
\mathbb{C}_{H A}=3 k_{H A} \mathbb{I}^{v o l}+2 \mu_{H A} \mathbb{I}^{d e v}
$$

with $k_{H A}=82.6 \mathrm{GPa}$ and $\mu_{H A}=44.9 \mathrm{GPa}, \mathbb{I}^{v o l}$ as the volumetric part of the identity tensor $\mathbb{I}$ with components

$$
I_{i j k l}^{v o l}=\frac{1}{3} \delta_{i j} \delta_{k l}
$$

and $\mathbb{I}^{d e v}$ as the deviatoric part of the identity tensor defined by $\mathbb{I}^{d e v}=\mathbb{I}-\mathbb{I}^{\text {vol }}$ The elastic properties of the (undrained) pore fluid are approximated by that of the water: $\mathbb{C}_{\text {pore }}=3 k_{\mathrm{H}_{2} \mathrm{O}} \mathbb{I}^{\text {vol }}$, with $k_{\mathrm{H}_{2} \mathrm{O}}=2.3 \mathrm{GPa}$.

The strength properties of hydroxyapatite, as defined through the two parameters of the Mohr-Coulomb criterion, are gained from tension and compression experiments on different HA biomaterials (Peelen et al., 1978; Akao et al., 1981; Shareef et al., 1993), as given in greater detail in (Fritsch et al., 2009a). The Mohr-Coulomb parameters are chosen so as to minimize the mean square error between model predictions (as functions of porosity) and corresponding experimental results. They amount to: $\beta=12$ and $\sigma_{y}=570 \mathrm{MPa}$, OR, ACCORDING TO EQ. (92) AND (93), $c_{\text {solid }}=82.2 \mathrm{MPA}$ AND $\varphi_{\text {solid }}=57.8^{\circ}$.

Finally, CONCERning the volume FraCtions, MCCARThy ET AL. (1990), FOR THE THIRD EQUINE METACARPUS, REPORT 35 VALUES EACH FOR THE MACROSCOPIC MASS DENSITIES $\rho_{\text {macro }}$, AND FOR THE VASCULAR POROSITIES $f_{\text {vas }}$, GIVING ACCESS TO THE EXTRAVASCULAR MASS DENSITIES AS

$$
\rho_{\text {exvas }}=\frac{\rho_{\text {macro }}-\rho_{\mathrm{H}_{2} \mathrm{O}} \times f_{\text {vas }}}{1-f_{\text {vas }}}
$$

SeE TABle 5 For corresponding numerical Results. Then, ReCENT MICROCT INVESTIGATIONS REVEALING A LACUNAR-CANALICULAR POROSITY PER EXTRAVASCULAR SPACE OF $f_{\text {lactcan }}^{\text {exvas }}=2.06 \%$ ARE CONSIDERED (SCHNEIDER ET AL., 2007, 2011), SO AS TO TRANSFORM THESE EXTRAVASCULAR MASS DENSITIES TO THE EXTRACELLULAR LEVEL, YIELD- 
ING THE EXTRACELLULAR MASS DENSITIES ACCORDING TO

$$
\rho_{\text {excel }}=\frac{\rho_{\text {exvas }}-\rho_{\mathrm{H}_{2} \mathrm{O}} \times f_{\text {lact }+ \text { can }}^{\text {exvas }}}{1-f_{\text {lac }+ \text { can }}^{\text {exvas }}}
$$

SEe TABle 5 FOR CORRESPONDING Numerical RESUlts. These UltraSTRUCTURAL MASS DENSITIES THEN ENTER THE "UNIVERSAL" COMPOSITION RULES OF VuONG AND HELlmich (2011), WHO SHOWED that THE COMPOSITION OF THE EXTRACELLULAR BONE MATRIX, IN TERMS OF MINERAL, ORGANICS AND FLUID, FOLLOWS BILINEAR LAWS, AS BECAME EVIDENT FROM A WIDE VARIETY OF BONE TISSUES TESTED IN OVER 80 YeARS of RESEARCH (HAMmet, 1925; Burns, 1929; Gong ET AL., 1964; Biltz ANd Pellegrino, 1969; LeEs ET Al., 1979, 1983; Lees, 1987; Lees And Page, 1992; Lees et Al., 1995; Lees, 2003). These Rules are Depicted in Figure 8, Where the maCroscopiCTO-ULTRASTRUCTURAL MASS DENSITY TRANSITION WAS BASED ON VASCULAR POROSITIES OF $3 \%$ (LEES ET AL., 1979), AND LACUNAR-CANALICULAR POROSITIES OF $2 \%$ (SCHNEIDER ET AL., 2007, 2011) IN THE CASE OF MAMMALIAN BONE TISSUES, WHILE THE ABSENCE OF SUCH PORES IN THE CASE OF TENDONS AND OTIC BONE TISSUES DOES NOT REQUIRE DISCRIMINATION BETWEEN MACROSCOPIC AND ULTRASTRUCTURAL DENSITY AND CONCENTRATION PROPERTIES. THE EXTRACELLULAR BONE MATRIX IS MADE UP BY AN ARRANGEMENT OF COLLAGENEOUS FIBRILS AND COLLAGEN-DEFICIENT EXTRAFIBRILlAR SPACE, THESE TWO COMPARTMENTS BEING CHARACTERIZED BY THE SAME EXTRACOLLAGENEOUS mineral concentration (Hellmich and Ulm, 2003). This distribuTION RULE, TOGETHER WITH THE RULES IDENTIFIED BY MORIN ET AL. (2013) AND MORIN AND HELlmich (2013) FOR FIBRILlar sWELling AND SHRINKAGE DUE TO HYDRATION AND MINERALIZATION, FINALLY ALLOWS FOR QUANTIFICATION OF THE INTERCRYSTALLINE POROSITY AND THE MINERAL VOLUME FRACTIONS INSIDE AN RVE OF EXTRAFIBRILLAR SPACE, The one DepiCted in Figure 7(D); SEe TABle 5 For CORRESPONDING RESULTS.

USE OF THESE VOLUME FRACTIONS FOR SUCH AN RVE SUBJECTED TO PURE SHEAR UNTIL FULL PLASTIFICATION, YIELDS THE SHEAR STRENGTH Values Given in the last COlumn of Table 5. Their mean value OF 31 MPA VIRTUALLY PERFECTLY AGREES WITH THE MEAN EXPERIMENTAL VAlue OF 30.7 MPA GIVEN By Bigley ET AL. (2006) FOR DEBOND SHEAR STRENGTH OF CEMENT LINES. 


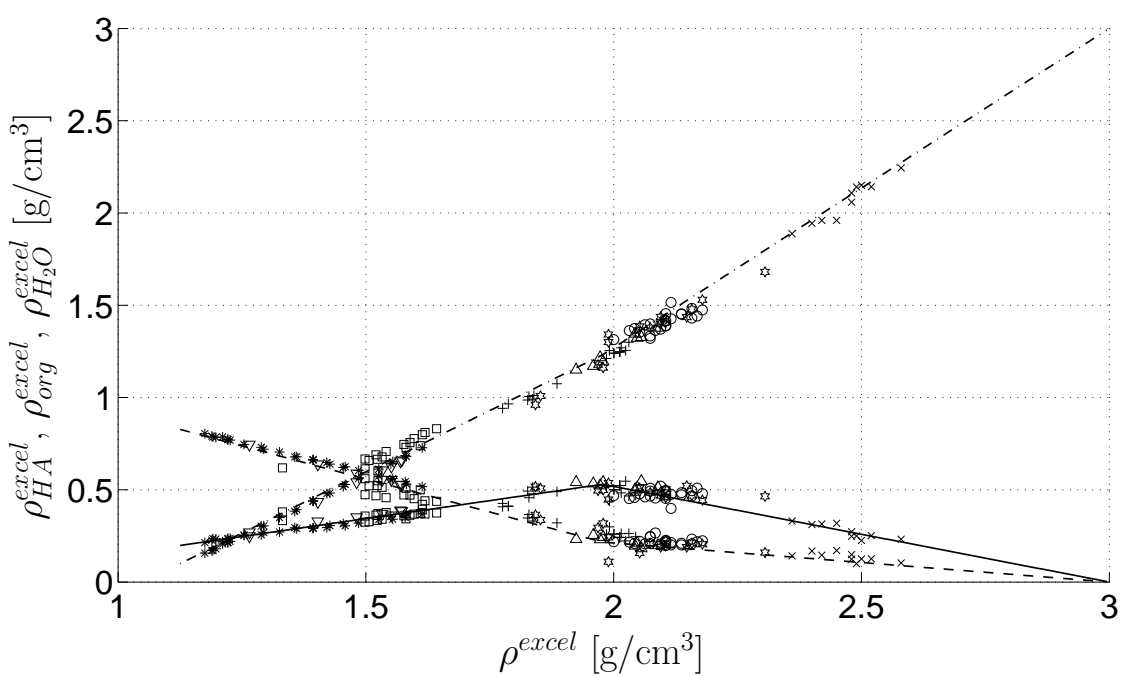

- Bovine bone (Lees et al., 1979)

× Otic bones of a fin whale (Lees et al., 1995)

- Mineralized turkey leg tendon (Lees and Page, 1992)

$\Delta$ Bones from steers, dogs, humans, monkeys (Gong et al., 1964)

$\nabla$ Bones from rabbits, rats (Burns, 1929)

* Bones from various vertebrates (Biltz and Pellegrino, 1969)

+ Horse metacarpals (Lees, 2003)

* Femur and humeris of albino rats (female and male) (Hammett, 1925)

- Organic

-. Mineral

- - - Water

Figure 8: APPARENT MASS DENSITIES (PER VOLUME OF EXTRACELLULAR SPACE) OF WATER, HYDROXYAPATITE, AND ORGANIC MATTER, VERSUS OVERALL MASS DENSITY OF EXTRACELLULAR BONE MATRIX, ACROSS DIFFERENT SPECIES, ORGANS, AND AGES; IN THE LINE OF VUONG AND HELLMiCH (2011) 
Table 5: Characterization OF EQUine CORTICAL BONE SAMPLES OF MCCARTHY ET AL. (1990): EXPERIMENTALLYDETERMINED MACROSCOPIC MASS DENSITIES AND VASCULAR POROSITIES; EXTRAVASCULAR AND EXTRACELLULAR MASS DENSITIES ACCORDING TO EQ. (99) AND (100); VOLUME FRACTIONS OF HYDROXYAPATITE CRYSTAL NEEDLE PHASE AND OF PORE PHASE ACCORDING TO "UNIVERSAL" COMPOSITION RULES REPORTED IN (VUONG AND Hellmich, 2011; Hellmich AND Ulm, 2003; Morin Et Al., 2013; Morin and Hellmich, 2013); AND Shear STRENGTH VALUES PREDICTED BY THE MICROMECHANICS MODEL FROM SECTION 6, BASED ON THE VOLUME FRACTIONS AND THE MATERIAL PROPERTIES OF TABLE 3.

\begin{tabular}{c|c|c|c|c|c|c}
$\begin{array}{c}\rho_{\text {macro }} \\
{\left[\mathrm{g} / \mathrm{cm}^{3}\right]}\end{array}$ & $\begin{array}{c}f_{\text {vas }} \\
{[-]}\end{array}$ & $\begin{array}{c}\rho_{\text {exvas }} \\
{\left[\mathrm{g} / \mathrm{cm}^{3}\right]}\end{array}$ & $\begin{array}{c}\rho_{\text {excel }} \\
{\left[\mathrm{g} / \mathrm{cm}^{3}\right]}\end{array}$ & $\begin{array}{c}f_{H A} \\
{[-]}\end{array}$ & $\begin{array}{c}f_{\text {pore }} \\
{[-]}\end{array}$ & $\begin{array}{c}\sum_{23}^{\text {ult }} \\
{[\mathrm{MPa}]}\end{array}$ \\
\hline 2.03 & 0.10 & 2.14 & 2.17 & 0.74 & 0.26 & 32.5 \\
2.02 & 0.08 & 2.11 & 2.13 & 0.72 & 0.28 & 31.7 \\
2.01 & 0.11 & 2.13 & 2.16 & 0.74 & 0.26 & 32.2 \\
2.01 & 0.07 & 2.09 & 2.11 & 0.71 & 0.29 & 31.2 \\
2.00 & 0.09 & 2.10 & 2.12 & 0.72 & 0.28 & 31.5 \\
2.00 & 0.07 & 2.08 & 2.10 & 0.71 & 0.29 & 31.0 \\
2.00 & 0.06 & 2.06 & 2.09 & 0.70 & 0.30 & 30.7 \\
1.98 & 0.12 & 2.11 & 2.14 & 0.73 & 0.27 & 31.8 \\
1.98 & 0.12 & 2.11 & 2.14 & 0.73 & 0.27 & 31.8 \\
1.98 & 0.10 & 2.09 & 2.11 & 0.71 & 0.29 & 31.3 \\
1.98 & 0.10 & 2.09 & 2.11 & 0.71 & 0.29 & 31.3 \\
1.97 & 0.10 & 2.08 & 2.10 & 0.71 & 0.29 & 31.0 \\
1.97 & 0.12 & 2.10 & 2.13 & 0.72 & 0.28 & 31.6 \\
1.96 & 0.11 & 2.08 & 2.10 & 0.71 & 0.29 & 31.0 \\
1.96 & 0.10 & 2.07 & 2.09 & 0.70 & 0.30 & 30.8 \\
1.95 & 0.14 & 2.10 & 2.13 & 0.72 & 0.28 & 31.6 \\
1.95 & 0.09 & 2.04 & 2.07 & 0.69 & 0.31 & 30.3 \\
1.95 & 0.12 & 2.08 & 2.10 & 0.71 & 0.29 & 31.1 \\
1.95 & 0.18 & 2.16 & 2.18 & 0.75 & 0.25 & 32.7 \\
1.95 & 0.11 & 2.07 & 2.09 & 0.70 & 0.30 & 30.8 \\
1.95 & 0.14 & 2.10 & 2.13 & 0.72 & 0.28 & 31.6 \\
1.93 & 0.12 & 2.06 & 2.08 & 0.70 & 0.30 & 30.6 \\
1.93 & 0.09 & 2.02 & 2.04 & 0.67 & 0.33 & 29.5 \\
1.93 & 0.13 & 2.07 & 2.09 & 0.70 & 0.30 & 30.9 \\
1.92 & 0.12 & 2.05 & 2.07 & 0.69 & 0.31 & 30.4 \\
1.92 & 0.10 & 2.02 & 2.04 & 0.67 & 0.33 & 29.6 \\
1.92 & 0.12 & 2.05 & 2.07 & 0.69 & 0.31 & 30.4 \\
1.92 & 0.11 & 2.03 & 2.06 & 0.69 & 0.31 & 30.0 \\
1.91 & 0.12 & 2.03 & 43.06 & 0.69 & 0.31 & 30.0 \\
1.91 & 0.22 & 2.17 & 2.19 & 0.75 & 0.25 & 32.9 \\
1.91 & 0.18 & 2.11 & 2.13 & 0.72 & 0.28 & 31.7 \\
1.90 & 0.25 & 2.20 & 2.23 & 0.77 & 0.23 & 33.5 \\
1.90 & 0.12 & 2.02 & 2.04 & 0.68 & 0.32 & 29.6 \\
1.82 & 0.09 & 1.90 & 1.92 & 0.57 & 0.43 & 24.9 \\
1.76 & 0.30 & 2.09 & 2.11 & 0.71 & 0.29 & 31.2 \\
& & & & & &
\end{tabular}


WHILE THIS IMPRESSIVELY CONFIRMS OUR MODELING APPROACH, WE WISH TO ALSO BASE THE EXPERIMENTAL VALIDATION OF THE MODELPREDICTED VALUES OF TABLE 5 ON A MORE PROFOUND STATISTICAL ARGument. Therefore, WE CONSIDER The AFOREMENTIONED STRENGTH PREDICTIONS AS VALIDATED IF AND ONLY IF THE FOLLOWING STATISTICAL QUESTION IS ANWERED With "YeS". The QUeSTiOn IS: Does The STATISTICAL SAMPLE OF 35 MODEL-PREDICTED SHEAR STRENGTH VALUES BELONG TO THE SAME STATISTICAL POPULATION OF SHEAR STRENGTH VALUES AS THE STATISTICAL SAMPLE OF 66 STRENGTH VALUES WHICH WERE EXPERIMENTALLY OBTAINED By Bigley ET AL. (2006)? IF WE RECONSTRUCT THE LATTER STATISTICAL SAMPLE OF Bigley ET AL. (2006) THROUGH LOG-NORMALLY DISTRIBUTED RANDOM VARIABLES WITH A MEAN VALUE OF 30.7 MPA AND A STANDARD DEVIATION OF 3.9 MPA, I.E. WiTH THE CHARACTERISTICS GIVEN EXPLICITLY BY Bigley ET AL. (2006), THEN AN ANOVA TEST CLEARLY PROVIDES A CLEAR "YES" TO THE AFOREMENTIONED QUESTION (FOR 5 RANDOMLY RECONSTRUCTED EXPERIMENTAL SAMPles, THE CORRESPONDING $F$-VALUES, WITH A MEAN OF 0.49, STAYS VERY CLEARLY BELOW THE CRITICAL $F$-VALUE OF 3.94 ). CONSEQUENTLY, WE REGARD OUR MICROMECHANICAL MODEL OF PLASTIC POROUS POLYCRYSTALS AS VALIDATED FOR CEMENT LINES IN OSTEONAL BONE.

\section{Summary and conclusions}

While multiscale elastoplasticity remains a veritable challenge in the mechanics and physics of solids, the present paper contains several original contributions providing a computationally efficient way to compute plastic phenomena in porous hydrated polycrystals:

- The influence tensor concept pioneered by Dvorak and colleagues (Dvorak and Benveniste, 1992; Dvorak, 1992; Dvorak et al., 1994) has been extended to infinitely many, mutually interacting, NEEDLE-SHAPED phases oriented in all space directions. This appears as a valuable solution for elastoplastic upscaling in polycrystals; which overcomes the elastoplastic stiffness overestimation associated with traditional micromechanics approaches comprising typically only a few (such as two) phases with (assumedly) uniform plastic strains; see e.g. the discussions in (Chaboche et al., 2001, 2005; Shojaei and Li, 2013). Similarly 
effective solutions have been already proposed in the context of the socalled NTFA - non-uniform transformation field analysis (Michel and Suquet, 2003, 2004; Roussette et al., 2009; Jiang et al., 2013; Fritzen and Böhlke, 2011); however, the latter approaches have all been related to periodic (rather than polycrystalline) microstructures, and the determination of associated plastic modes (replacing uniform plastic strain fields) typically requires the introduction of coupled plastic flow rules and auxiliary numerical simulations, based on the Finite Element, or the Fast Fourier Transform Method. As with the Finite Element Method, also our present approach needs consideration of discretization issues (related, however, to the proper evaluation of integrals over the unit sphere), while it is based on classical multisurface plasticity (with no need to introduce additional plastic couplings).

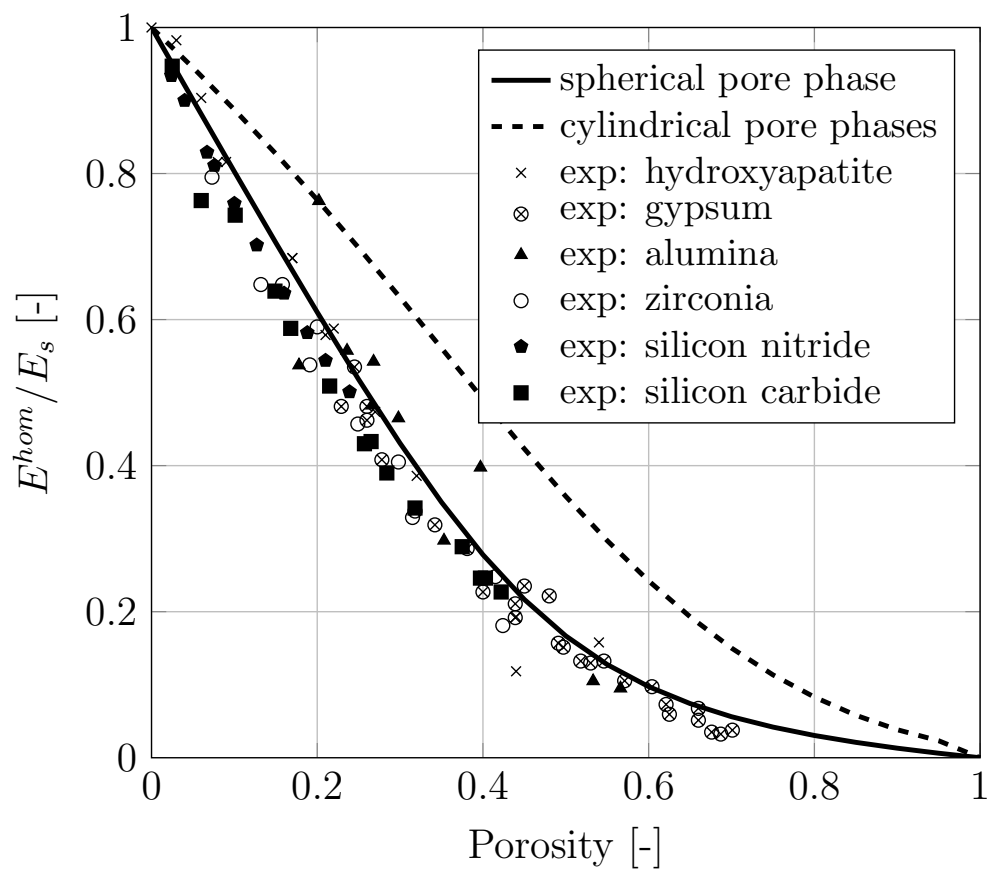

Figure 9: COMPARISON OF SPHERICAL AND CYLINDRICAL PORE SHAPE REPRESENTATION IN MICROMECHANICAL MODEL FOR POROUS POLYCRYSTAL; AND COMPARISON WITH EXPERIMENTS, SEE (FRITSCH ET AL., 2013) FOR EXPERIMENTAL SOURCES. 
- The morphology depicted in Figure 1 is Particularly Well SUITED FOR A WIDE ClASS OF POROUS POLYCRYSTALS. THIS WAS ShOWN BY Fritsch ET AL. (2009B); SANAhUja ET AL. (2010); FRITSCH ET AL. (2013); REPORTING THAT THE HOMOGENIZED YOUNG'S MODULUS NORMALIZED BY THE YOUNG'S MODULUS OF THE SOLID NEEDLE PHASES TURNED OUT TO EXCLUSIVELY DEPEND ON THE POROSITY, AND THAT THIS DEPENDENCE WAS VERY WELL CONFIRMED BY EXPERIMENTAL DATA ON HYDROXYAPATITE (DE WITH ET AL., 1981; Gilmore And Katz, 1982; LiU, 1998; Charrière et Al., 2001), GyPSUm (Ali And Singh, 1975; Phani, 1986; Tazawa, 1998; Meille, 2001; Colak, 2006), Piezoelectric CeRAMics (Craciun ET AL., 1998), Alumina (Coble And Kingery, 1956; PABst et Al., 2004), ZirConia (PABSt ET AL., 2004), SilICON CARBIDE (REYNAUD ET AL., 2005), AND SILICON NITRIDE (DÍAz AND HAMPSHIRE, 2004). In ADDITION, SUCH HOMOGENIZED ELASTICITY PREDICTIONS WERE SHOWN TO BE IN EXCELLENT AGREEMENT With Finite Element Simulations of "REAL" GYPSUM MICrostructures (SAnahuja et Al., 2010). By COMPARISOn, APPROACHES WHICH MAY SEEM SIMILARLY ADEQUATE ON THE FIRST SIGHT, SUCH AS THE USE OF UNIFORMLY ORIENTED ACIRCULAR PORE PHASES INSTEAD OF ONE SPHERICAL PORE PHASE, YIELD REMARKABLY WORSE PREDICTIONS, NAMELY A SIGNIFICANT OVEREStimation of EXPERIMENTAL DATA, SEe Figure 9. Further CONFIRMING THE CHOICE OF ONE SPHERICAL PORE PHASE, THE MICROMECHANICAL REPRESENTATION OF FIgURE 1 ALSO TURNED OUT AS VERY RELEVANT FOR QUASI-BRITTLE STRENGTH PREDICTiOnS (Fritsch ET AL., 2009B; SANAHUJA ET AL., 2010); AND THE PRESENT PAPER SHOWS AN EQUALLY SOUND CAPACITY OF THIS MICROMECHANICAL REPRESENTATION WHEN IT COMES TO THE REALM OF ELASTOPLASTIC PROPERTY UPSCALING. EXTENSION OF THE METHOD FROM UNIFORMLY ORIENTED NEEDLE-SHAPED PHASES TO PREFERENCE OF CERTAIN ORIENTATIONS, AS ENCOUNTERED E.G. IN THE CASE OF CLAY (WENK ET AL., 2008) IS QUITE STRAIGHTFORWARD. AS WAS SHOWN FOR AXISYMMETRICALLY ORIENTED NEEDLE PHASES IN THE CONTEXT OF ELASTICITY (FRITSCH ET AL., 2006), AND WOULD BE REFLECTED BY ADDING PROBABILITY DENSITY FUNCTIONS INSIDE THE SPHERICAL INTEGRALS APPEARING IN EQS. (16), (17), (22), (23), (25), (26), (31), (32), (33), (34), (35), 
(38), (39), (40), (41), (42), (43) AS WELL AS IN THEIR DISCRETIZED COUNTERPARTS. THIS IS BEYOND THE SCOPE OF THE PRESENT MANUSCRIPT.

- Involving infinitely many phases as part of the basic concept, our approach requires discretization down to an approriate number of phases, for which we employ two different concepts, namely Stroud's integration (Stroud, 1971) as well as the discretization method described in (Badel and Leblond, 2004). The results related to all three choices of discretization are in satisfactory agreement. In this context, we reiterate that the consideration of different orientations is one of the keys to the succcess of our new method, a feature which this method shares with the so-called microplane models, as developed extensively by Bažant and colleagues (Bažant and Oh, 1985; Bažant and Prat, 1988; Bažant et al., 2000). The latter models are also based on the principle of virtual work, however, they restrict corresponding energetical considerations to tangential planes on a unit hemisphere; rather than to a $3 \mathrm{D}$ RVE as is typically done in continuum micromechanics and described in Section 2.

- Upscaling of the elastoplastic constitutive relations from the solid phase to the porous polycrystal scale is performed by adaptation of the wellknown return map algorithm originating from the nonlinear elastoplastic Finite Element analysis (Simo and Hughes, 1998; Simo and Taylor, 1985), based on an incremental load apposition. What is adopted from the original algorithm is the trial state computed from a fictitiously purely elastic deformation in the load step under investigation; this trial step giving a first indication on which solid phases might actually undergo plastic deformations. Whether this is actually the case, is checked via implicit solution of the elastic constitutive equation in combination with fulfillment of the active yield criteria. In contrast to the case encountered in the nonlinear Finite Element analysis, computation of elastoplastic (consistent) tangent is not required, as the concentration-influence relations are explicitly known. We regard this adoption of classical plasticity algorithms for micromechanics approaches as original in the context of polycrystalline materials, while we are aware of similar approaches for metal-ceramic composites with periodic microstructures (Vena et al., 2007); BUT ALSO FOR 


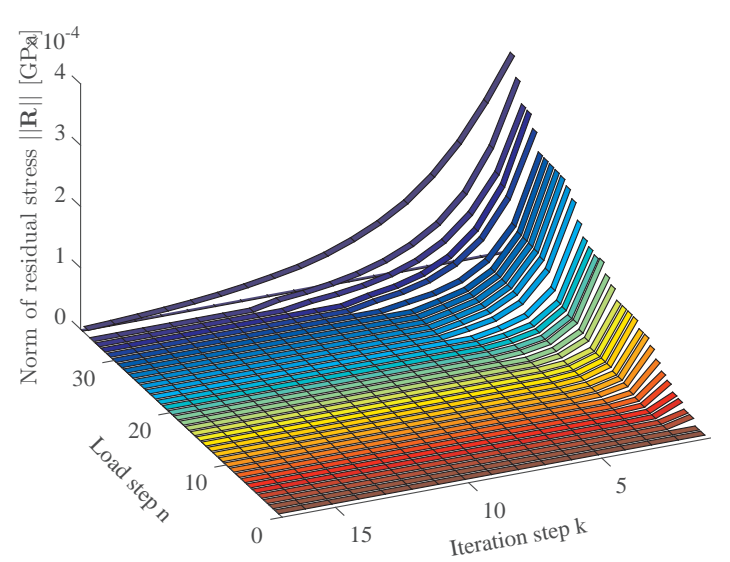

(a) under pure shear

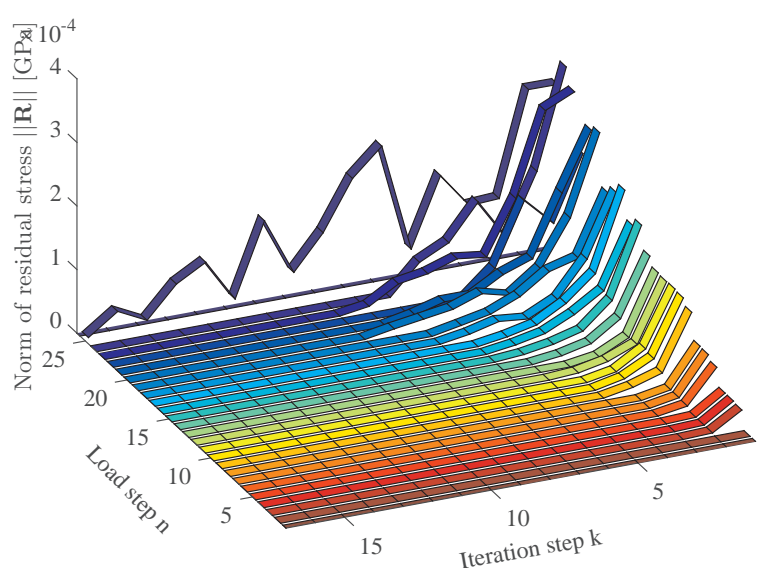

(b) under uniaxial tension

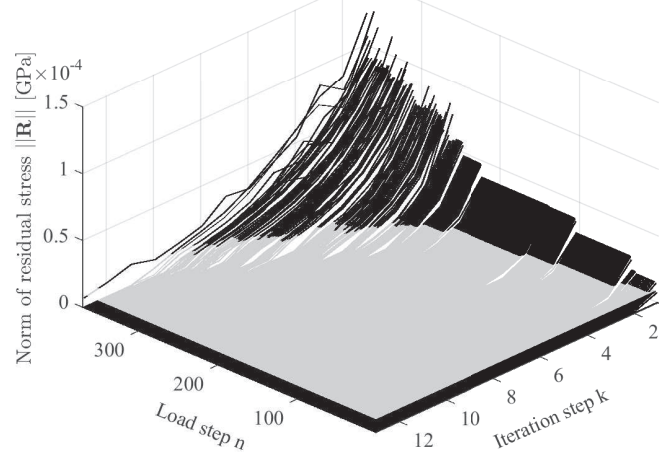

(c) under uniaxial compression

Figure 10: Convergence BeHAVIOR DURING RETURN MAP OF TRIAL STATES ONTO MOHR-COULOMB SURFACES RELATED TO 120 SOLID NEEDLE PHASES ARISING FROM BADELLEBLOND DISCRETIZATION; RESIDUAL VECTOR $\mathbf{R}$ IS DEFINED THROUGH EQ. (84) 
SELF-CONSISTENT HOMOGENIZATION BASED ON SPHERICAL PHASE REPRESENTATIONS (ZENG ET AL., 2014). THE LATTER REFERENCE, HOWEVER, RESTS ON THE USE OF SUITABLY CHOSEN ELASTOPLASTIC TANGENTS, WHILE OUR CONCEPT EXPLICITLY ACCOUNTS FOR THE EFFECTS OF GEOMETRICALLY INCOMPATIBLE NON-ELASTIC STRAINS AT THE MICROSCALE, ON THE OVERALL HOMOGENIZED MATERIAL BEHAVIOR, EXTENDING THE TRANSFORMATION FIELD ANALysis (DvoraK AND Benveniste, 1992; DvoraK Et AL., 1994), BASEd on MORE RECENT WORK of PiChler ANd Hellmich (2010), TOWARDS INFINITELY MANY, NEEDLE-SHAPED EIGENSTRESSED PHASES. ReAlization of a RETURN MAP ALGORITHM FOR MOHR-COUlOMB PLASTICITY IS A QUITE PECULIAR UNDERTAKING. OUR CORRESPONDING DEVELOPMENTS DESCRIBED IN SECTION 6 ARE SOMEWhat INSPIRED BY THE WORK OF Clausen ET AL. (2007). HowEVER, THE LATTER WORK CONSIDERS CASES WHERE THE PRINCIPAL STRESS DIRECTIONS STAY UNALTERED DURING THE BACK PROJECTION OF THE TRIAL STRESS STATE (WHICH IS RELATED TO ONE POINT OF THE SIMULATED STRUCTURE), ONTO THE CORRESPONDing yiEld surface. Hence, this RETURn mapPing Algorithm, WHICH IS PERFORMED STRESS STATE PER STRESS STATE, IS ASSOCIATED WITH THE SOLUTION OF A LINEAR SYSTEM OF EQUATIONS. WiTH RESPECT TO THIS SITUATION, OUR SOLUTION DESCRIBED IN SECTION 5.4 IS ORIGINAL WITH RESPECT TO TWO FEATURES: (I) ALL TRIAL STRESS STATES IN ALL (ACTIVE) NEEDLE PHASES ARE BACK PROJECTED SIMULTANEOUSLY, LEADING FINALLY TO A MUCH LARGER SYSTEM OF EQUATIONS INVOLVING ALL PLASTIC MULTIPLIERS FROM ALL YIELD SURFACES RELATED TO ALL (ACTIVE) NEEDLE PHASES; AND (II) THE PRINCIPAL STRESS DIRECTIONS change UPON BACK PROJECTION OF THE TRIAL STRESS STATES ONTO THE PLASTIC YIELD SURFACES; ACCORDINGLY THE AFOREMENTIONED SYSTEM OF EQUATIONS BECOMES nonlinear, AND IT IS HENCE SOLVED ACCORDING TO THE ITERATION SCHEME GIVEN BY EQ. (82) - (88). DEPENDING ON MACROSCOPIC LOADING TYPE AND LOADING LEVEL, I.E. ON THE NUMBER OF ACTIVE NEEDLE-SHAPED SOLID PHASES, BETWEEN ONE AND TEN ITERATION STEPS ARE TYPICALLY NEEDED IN ORDER TO BACK-PROJECT ALL TRIAL STRESS STATES ONTO THE PHASE-SPECIFIC YIELD SURFACES, SEE FiguRES 10(A)-(C). 
- As a rule the microstructural plastic events start in crystal phases oriented in the direction of the dominant macroscopic tensile stress; in the case of the latter being absent, the plasticity affects solid phases being oriented perpendicular to the dominant macroscopic compressive direction. Thereafter, the micro-plasticity spreads over all solid phase needle directions. Ultimate loads refer to all solid needle plasticizing, and when applied to the collagen-poor lamellae of osteonal bone, the presented micromechanical concept predicts well the ultimate loads attained in osteon push-out tests. Corresponding Mohr-Coulomb PLASTICITY OF THE HYDROXYPATITE CRYSTAL PHASES THEREBY MAY REPRESENT EITHER SLIDING OF HYDRATED INTERFACES SITUATED WITHIN THESE PHASES AS REPEATEDLY DISCUSSED IN MATERial mechanics (Bhowmik et Al., 2007, 2009; Shahidi et AL., 2014; QU ET AL., 2015A,B) OR INTRINSIC DISLOCATION PHENOMENA IN HYdROXYAPATITE (ViSWANATH ET AL., 2007; SAKA ET AL., 2008; IEVLEV ET AL., 2013).

- As regards model VAlidation, there are tWo PRINCIPAl OPTIONS: COMPARISON OF MICROMECHANICS MODEL RESULTS WITH Finite ElEMENT SIMULATIONS RESOLVING THE MATERIAL MICROSTRUCTURES DOWN TO MINUTE DETAIL; OR WITH EXPERIMENTAL REsults. Generation of trustworthy Finite Element modELS REQUIRES VERY DETAILED KNOWLEDGE ON THE GEOMETRICAL CHARACTERISTICS OF THE OBJECT TO BE SIMULATED. AS REGARDS THE EXTRAFIBRILLAR SPACE OF BONE, THE MATERIAL SYSTEM INVESTIGATED IN SECTION 7, TRANSMISSION ELECTRON Micrographs (Lees And Prostak, 1988; Lees et AL., 1994; Prostak and Lees, 1996; Zylberberg et Al., 1998; Su et Al., 2003; MCNally ET AL., 2012) haVE REVEALED MiCROSTRUCTURAL DETAILS DOWN TO THE SEVERAL NANOMETER SCALE, OVER RANGES SPANNING SEVERAL HUNDREDS OF NANOMETERS. HOWEVER, FULL 3D QUANTIFICATION OF STRUCTURAL FEATURES, E.G. BY MEANS OF X-RAY-Based COMPUted TOMOgRAPHy TeCHNiques Which HAVE PROVED AS PARTICULARLY USEFUL FOR THE GENERATION OF Trustworthy Finite Element models (Dejaco ET AL., 2012), IS STILL OUT OF REACH. THIS IS WHY WE HERE COMPARE OUR HOMOGENIZATION RESUlTS OF TABLE 5 DIRECTLY TO THE EXPERIMENTAL RESUlts OF Bigley ET AL. (2006) FOR DEBONDING SHEAR 
STRENGTH OF CEMENT LINES: MODEL PREDICTIONS AND EXPERIMENTS AGREE VIRTUALLY PERFECTLY, AS REPORTED IN THE LAST PARAGRAPH OF SECTION 7. FROM A MORE QUALITATIVE VIEWPOINT, IT IS INTERESTING THAT THE SEAMLESS TRANSITION FROM ELASTIC TO ELASTOPLASTIC REGIMES IN THE STRESS-STRAIN CURVES OF BONE AT THE SINGLE MICRON SCALE, WHERE POLYCRYSTALS AS THE ONE DEPICTED IN FiguRE 1 LARGELY AFFECT THE MATERIAL BEHAVIOR, IS INDEED OBSERVED EXPERIMENTALLY, AS RECENT COMPRESSIVE TESTS ON SINGLE MICRON-SIZED PILLARS HAVE IMPRESSIVELY SHOWN (SCHWIEDRZIK ET AL., 2014; LUCZYNSKI ET AL., 2015).

- For the sake of completeness, we may state that our apPRoAch targets at CAPTURING THE DEVElopMENT OF MiCROPLASTIC STRAINS STEMMING FROM NON-ASSOCIATED PLASTICITY, AND THEIR MACROSCOPIC EFFECTS. THIS MAY BE SEEN AS AN INTERESTING COMPLEMENT TO THE POPULAR PLASTIC HOMOGENIZATION APPROACHES BASED ON LIMIT ANALYSIS AND VARIATIONAL METHODS; CONSIDERING, AS A RULE, MICROSCOPIC STRENGTH CRITERIA, EQUILIBRIUM AT THE MICRO AND MACRO-SCALE, MAXIMIZATION OF DISSIPATION, AND SOMETIMES ASSOCIATED PLASTICITY. Corresponding RECEnT DEVElopments ARE REPORTED IN (Cheng et Al., 2014; Shen et Al., 2015; Bignonnet et Al., 2016).

- Potential future work may aim at going Beyond the CONSTRAINTS OF IDEAL PLASTICITY AT THE SOLID NEEDLE PHASE LEVEL, SO AS TO EXTEND THE MODELING APPROACH TOWARDS CATASTROPHIC FAILURE, SUCH AS IT IS EVENTUALLY SEEN, FOR EXAMPLE, IN THE Osteon Push-OUt TeSts of (Bigley ET AL., 2006). This WOULD IMPLY CAREFUL STUDY OF SO-CALLED SOFTENING PLASTICITY FORMULATIONS, CONSIDERING VALUABLE KNOWLEDGE FROM NONLOCAL AND GRADIENT PLASTICITY THEORY (DE SCIARRA, 2004, 2008A,B, 2009).

\section{Acknowledgments}

Financial support in the framework of project ERC-2010-StG-257023MICROBONE is gratefully acknowledged. The cooperation between TU Wien and EMSE was facilitated by the bilateral Hubert Curien - Amadeus 
travel grant FR02/2015 of OEAD - Austrian Agency for International Cooperation in Education and Research and Campus France - French Agency for International Cooperation in Education and Research. 


\section{References}

Akao, M., Aoki, H., Kato, K., 1981. Mechanical properties of sintered hydroxyapatite for prosthetic applications. Journal of Materials Science 16 (3), 809-812.

Ali, M., Singh, B., 1975. The effect of porosity on the properties of glass fibrereinforced gypsum plaster. Journal of Materials Science 10 (11), 1920-1928.

Ascenzi, A., Bonucci, E., 1972. The shearing properties of single osteons. The Anatomical Record 172 (3), 499-510.

Badel, P., Leblond, J., 2004. A note on integration schemes for the microplane model of the mechanical behaviour of concrete. Communications in Numerical Methods in Engineering 20 (1), 75-81.

Bažant, Z., Caner, F., Carol, I., Adley, M., Akers, S., 2000. Microplane model M4 for concrete. I: Formulation with work-conjugate deviatoric stress. Journal of Engineering Mechanics 126, 944-953.

Bažant, Z., Oh, B., 1985. Microplane model for progressive fracture of concrete and rock. Journal of Engineering Mechanics 111, 559-582.

Bažant, Z., Prat, P., 1988. Microplane model for brittle plastic material: I. Theory. Journal of Engineering Mechanics 114, 1672-1688.

Benveniste, Y., 1987. A new approach to the application of Mori-Tanaka's theory in composite materials. Mechanics of Materials 6 (2), 147-157.

Berveiller, M., Zaoui, A., 1978. An extension of the self-consistent scheme to plastically-flowing polycrystals. Journal of the Mechanics and Physics of Solids 26 (5-6), 325-344.

Bhowmik, R., Katti, K., Katti, D., 2007. Mechanics of molecular collagen is influenced by hydroxyapatite in natural bone. Journal of Materials Science 42 (21), 8795-8803.

Bhowmik, R., Katti, K., Katti, D., 2009. Mechanisms of load-deformation behavior of molecular collagen in hydroxyapatite-tropocollagen molecular system: Steered molecular dynamics study. Journal of Engineering Mechanics 135 (5), 413-421. 
Bigley, R., Griffin, L., Christensen, L., Vandenbosch, R., 2006. Osteon interfacial strength and histomorphometry of equine cortical bone. Journal of Biomechanics 39 (9), 1629-40.

Bignonnet, F., Dormieux, L., Kondo, D., 2016. A micro-mechanical model for the plasticity of porous granular media and link with the Cam clay model. International Journal of Plasticity 79, 259-274.

Biltz, R., Pellegrino, E., 1969. The chemical anatomy of bone. I. A comparative study of bone composition in sixteen vertebrates. The Journal of Bone and Joint Surgery 51 (3), 456-466.

Burns, C., 1929. The effect of the continued ingestion of mineral acid on growth of body and bone and on the composition of bone and of the soft tissues. Biochemical Journal 23 (5), 860-867.

Cavalcante, M., Pindera, M.-J., 2016. Generalized FVDAM theory for elasticeplastic periodic materials. International Journal of Plasticity 77, 90-117.

Cayzac, H.-A., Säi, K., Laiarinandrasana, L., 2013. Damage based constitutive relationships in semi-crystalline polymer by using multi-mechanisms model. International Journal of Plasticity 51, 47-64.

Chaboche, J., Kanouté, P., Roos, A., 2005. On the capabilities of mean-field approaches for the description of plasticity in metal matrix composites. International Journal of Plasticity 21 (7), 1409-1434.

Chaboche, J., Kruch, S., Maire, J., Pottier, T., 2001. Towards a micromechanics based inelastic and damage modeling of composites. International Journal of Plasticity 17 (4), 411-439.

Charrière, E., Terrazzoni, S., Pittet, C., Mordasini, P., Dutoit, M., Lemaître, J., Zysset, P., 2001. Mechanical characterization of brushite and hydroxyapatite cements. Biomaterials 22 (21), $2937-2945$.

Cheng, L., de Saxcé, G., Kondo, D., 2014. A stress-based variational model for ductile porous materials. International Journal of Plasticity 55, 133151.

Clausen, J., Damkilde, L., Andersen, L., 2007. An efficient return algorithm for non-associated plasticity with linear yield criteria in principal stress space. Computers and Structures 85 (23-24), 1795-1807. 
Coble, R., Kingery, W., 1956. Effect of porosity on physical properties of alumina. Journal of the American Ceramic Society 39, 377-385.

Colak, M., 2006. Physical and mechanical properties of polymer-plaster composites. Material Letters 60 (16), 1977-1982.

Constantinides, G., Ulm, F.-J., 2004. The effect of two types of C-S-H on the elasticity of cement-based materials: Results from nanoindentation and micromechanical modeling. Cement and Concrete Research 34 (1), 67-80.

Craciun, F., Galassi, C., Roncari, E., Filippi, A., Guidarelli, G., 1998. Electro-elastic properties of porous piezoelectric ceramics obtained by tape casting. Ferroelectrics 205, 49-67.

Davies, J., 2007. Bone bonding at natural and biomaterial surfaces. Biomaterials 28 (34), 5058-5067.

de Sciarra, F. M., 2004. Nonlocal and gradient rate plasticity. International Journal of Solids and Structures 41 (26), 7329 - 7349.

de Sciarra, F. M., 2008a. A general theory for nonlocal softening plasticity of integral-type. International Journal of Plasticity 24 (8), 1411 - 1439.

de Sciarra, F. M., 2008b. Variational formulations, convergence and stability properties in nonlocal elastoplasticity. International Journal of Solids and Structures 45 (78), $2322-2354$.

de Sciarra, F. M., 2009. Novel variational formulations for nonlocal plasticity. International Journal of Plasticity 25 (2), $302-331$.

de With, G., Van Dijk, H., Hattu, N., Prijs, K., 1981. Preparation, microstructure and mechanical properties of dense polycrystalline hydroxy apatite. Journal of Materials Science 16 (6), 1592 - 1598.

Dejaco, A., Komlev, V., Jaroszewicz, J., Swieszkowski, W., Hellmich, C., 2012. Micro CT-based multi scale elasticity of double-porous (pre-cracked) hydroxyapatite granules for regenerative medicine. Journal of Biomechanics 45 (6), 10681075.

Díaz, A., Hampshire, S., 2004. Characterisation of porous silicon nitride materials produced with starch. Journal of the European Ceramic Society $24(2), 413-419$. 
Doghri, I., Ouaar, A., 2003. Homogenization of two-phase elasto-plastic composite materials and structures: Study of tangent operators, cyclic plasticity and numerical algorithms. International Journal of Solids and Structures 40 (7), 1681-1712.

Dormieux, L., Kondo, D., Ulm, F.-J., 2006. Microporomechanics, 1st Edition. Wiley, Chichester, U.K.

Dvorak, G. J., 1992. Transformation field analysis of inelastic composite materials. Proceedings of the Royal Society A: Mathematical, Physical and Engineering Sciences 437 (1900), 311-327.

Dvorak, G. J., Bahei-El-Din, Y., Wafa, A., 1994. The modeling of inelastic composite materials with the transformation field analysis . Modelling and Simulation in Materials Science and Engineering 2 (3), 571-586.

Dvorak, G. J., Benveniste, Y., 1992. On transformation strains and uniform fields in multiphase elastic media. Proceedings of the Royal Society A: Mathematical, Physical and Engineering Sciences 437 (1900), 291-310.

Fritsch, A., Dormieux, L., Hellmich, C., 2006. Porous polycrystals built up by uniformly and axisymmetrically oriented needles: homogenization of elastic properties. Comptes Rendus Mécanique 334 (3), 151-157.

Fritsch, A., Dormieux, L., Hellmich, C., Sanahuja, J., 2009a. Mechanical behavior of hydroxyapatite biomaterials: An experimentally validated micromechanical model for elasticity and strength. Journal of Biomedical Materials Research, Part A 88A (1), 149-161.

Fritsch, A., Hellmich, C., 2007. Universal microstructural patterns in cortical and trabecular, extracellular and extravascular bone materials: Micromechanics-based prediction of anisotropic elasticity. Journal of Theoretical Biology 244 (4), 597-620.

Fritsch, A., Hellmich, C., Dormieux, L., 2009b. Ductile sliding between mineral crystals followed by rupture of collagen crosslinks: Experimentally supported micromechanical explanation of bone strength. Journal of Theoretical Biology 260 (2), 230-252. 
Fritsch, A., Hellmich, C., Young, P., 2013. Micromechanics-derived scaling relations for poroelasticity and strength of brittle porous polycrystals. Journal of Applied Mechanics 80 (2), 020905-1-12.

Fritzen, F., Böhlke, T., 2011. Nonuniform transformation field analysis of materials with morphological anisotropy. Composites Science and Technology 71 (4), 433-442.

Germain, P., 1973. The method of virtual power in continuum mechanics. Part 2: Microstructure. SIAM Journal on Applied Mathematics 25 (3), $556-575$.

Gilmore, R., Katz, J., 1982. Elastic properties of apatites. Journal of Materials Science 17 (4), 1131-1141.

Gong, J., Arnold, J., Cohn, S., 1964. Composition of trabecular and cortical bone. The Anatomical Record 149, 325-332.

Hammet, F., 1925. A biochemical study of bone growth. I Changes in the ash, organics matter and water during growth (mus norvegicus albinus). The Journal of Biological Chemistry 64, 409-428.

Hashin, Z., 1983. Analysis of composite materials - A survey. Journal of Applied Mechanics 50 (3), 481.

Hellmich, C., Ulm, F.-J., 2003. Average hydroxyapatite concentration is uniform in the extracollagenous ultrastructure of mineralized tissues: evidence at the 1-10-microm scale. Biomechanics and Modeling in Mechanobiology $2(1), 21-36$.

Hellmich, C., Ulm, F.-J., Mang, H., 1999. Multisurface chemoplasticity. I: Material model for shotcrete. Journal of Engineering Mechanics 125 (6), 692-701.

Hershey, A. V., 1954. The elasticity of an isotropic aggregate of anisotropic cubic crystals. Journal of Applied mechanics - Transactions of the ASME 21 (3), 236-240.

Hill, R., 1963. Elastic properties of reinforced solids: Some theoretical principles. Journal of the Mechanics and Physics of Solids 11 (5), 357-372. 
Hill, R., 1965a. A self-consistent mechanics of composite materials. Journal of the Mechanics and Physics of Solids 13 (4), 213-222.

Hill, R., 1965b. Continuum micro-mechanics of elastoplastic polycrystals. Journal of the Mechanics and Physics of Solids 13 (2), 89-101.

Ievlev, V., Kostyuchenko, A., Belonogov, E., Barinov, S., 2013. Hardness and the nature of microplasticity of hydroxyapatite. Inorganic Materials 49 (4), 416-422.

Jiang, T., Shao, J., Xu, W., 2013. A non-uniform transformation field analysis for frictional cohesive geomaterials. European Journal of Mechanics A/Solids 42, 97-111.

Katz, J., Ukraincik, K., 1971. On the anisotropic elastic properties of hydroxyapatite. Journal of Biomechanics 4 (3), 221-227.

Katz, J., Yoon, H., Lipson, S., Maharidge, R., Meunier, A., Christel, P., 1984. The effects of remodeling on the elastic properties of bone. Calcified Tissue International 36 (1), 3136.

Koiter, W., 1953. Stress-strain relations, uniqueness and variational theorems for elastic-plastic materials with a singular yield surface. Quarterly of Applied Mathematics 11, 350-354.

Kröner, E., 1958. Berechnung der elastischen Konstanten des Vielkristalls aus den Konstanten des Einkristalls. Zeitschrift für Physik 151 (4), 504-518.

Kruch, S., Chaboche, J.-L., 2011. Multi-scale analysis in elasto-viscoplasticity coupled with damage. International Journal of Plasticity 27, 2026-2039.

Laws, N., 1973. On the thermostatics of composite materials. Journal of the Mechanics and Physics of Solids 21 (1), 9-17.

Lees, S., 1987. Considerations regarding the structure of the mammalian mineralized osteoid from viewpoint of the generalized packing model. Connective Tissue Research 16 (4), 281-303.

Lees, S., 2003. Mineralization of type I collagen. Biophysical Journal 85, 204-207. 
Lees, S., Ahern, J., Leonard, M., 1983. Parameters influencing the sonic velocity in compact calcified tissues of various species. Journal of the Acoustical Society of America 74 (1), 28 - 33.

Lees, S., Cleary, P., Heeley, J., Gariepy, E., 1979. Distribution of sonic plesiovelocity in a compact bone sample. The Journal of the Acoustical Society of America 66 (3), 641-646.

Lees, S., Hanson, D., Page, E. A., 1995. Some acoustical properties of the otic bones of a fin whale. Journal of the Acoustical Society of America 99 (4), 2421-2427.

Lees, S., Page, E., 1992. A study of some properties of mineralized turkey leg tendon. Connective Tissue Research 28 (4), 263-287.

Lees, S., Prostak, K., 1988. The locus of mineral crystallites in bone. Connective Tissue Research 18 (1), $41-54$.

Lees, S., Prostak, K., Ingle, V., Kjoller, K., 1994. The loci of mineral in turkey leg tendon as seen by atomic force microscope and electron microscopy. Calcified Tissue International 55 (3), 180 - 189.

Levin, V., 1967. Thermal expansion coefficient of heterogeneous materials. Inzh. Zh. Mekh. Tverd. Tela 2 (1), 83-94.

Liu, D.-M., 1998. Preparation and characterisation of porous hydroxyapatite bioceramic via a slip-casting route. Ceramics International 24 (6), 441 446.

Luczynski, K., Steiger-Thrirsfeld, A., Bernardi, J., Eberhardsteiner, J., Hellmich, C., 2015. Extracellular bone matrix exhibits hardening elastoplasticity and more than double cortical strength: Evidence from homogeneous compression of non-tapered single micron-sized pillars welded to a rigid substrate. Journal of the Mechanical Behavior of Biomedical Materials $52,51-62$.

Marotti, G., 1993. A new theory of bone lamellation. Calcified Tissue International 53 (1), 47-56.

Marotti, G., Ferretti, M., Palumbo, C., 2013. The problem of bone lamellation: An attempt to explain different proposed models. Journal of Morphology 274 (5), 543-550. 
Marotti, G., Muglia, M., Palumbo, C., Zaffe, D., 1994. The microscopic determinants of bone mechanical properties. Italian Journal of Mineral and Electrolyte Metabolism 8 (4), 167-175.

McCarthy, R., Jeffcott, L., McCartney, R., 1990. Ultrasound speed in equine cortical bone: Effects of orientation, density, porosity and temperature. Journal of Biomechanics 23 (11), 1139-1143.

McNally, E., Schwarcz, H., Botton, G., Arsenault, A., 2012. A model for the ultrastructure of bone based on electron microscopy of ion-Milled sections. PLoS One 7, e29258.

Meille, S., 2001. Etude du comportement méanique du plâtre pris en relation avec sa microstructure (Study of the Mechanical Behaviour of Gypsum With Regard to its Microstructure). Ph.D thesis, INSA Lyon.

Michel, J., Suquet, P., 2003. Nonuniform transformation field analysis. International Journal of Solids and Structures 40 (2), 6937-6955.

Michel, J., Suquet, P., 2004. Computational analysis of nonlinear composite structures using the nonuniform transformation field analysis. Computer Methods in Applied Mechanics and Engineering 193 (48-51), 5477-5502.

Mori, T., Tanaka, K., 1973. Average stress in matrix and average elastic energy of materials with misfitting inclusions. Acta Metallurgica 21 (5), $571-574$.

Morin, C., Hellmich, C., 2013. Mineralization-driven bone tissue evolution follows from fluid-to-solid phase transformations in closed thermodynamic systems. Journal of Theoretical Biology 335, 185-197.

Morin, C., Hellmich, C., 2014. A multiscale poromicromechanical approach to wave propagation and attenuation in bone. Ultrasonics 54 (5), 12511269.

Morin, C., Hellmich, C., Henits, P., 2013. Fibrillar structure and elasticity of hydrating collagen: A quantitative multiscale approach. Journal of Theoretical Biology 317, 384-393.

Morin, C., Moumni, Z., Zaki, W., 2011a. A constitutive model for shape memory alloys accounting for thermomechanical coupling. International Journal of Plasticity 27 (5), $748-767$. 
Morin, C., Moumni, Z., Zaki, W., 2011b. Thermomechanical coupling in shape memory alloys under cyclic loadings: Experimental analysis and constitutive modeling. International Journal of Plasticity 27 (12), 1959 1980.

Nemat-Nasser, S., Hori, M., 1999. Micromechanics: overall properties of heterogeneous materials, 2nd Edition. Elsevier, Amsterdam, The Netherlands.

Pabst, W., Gregorová, E., Tichá, G., Týnová, E., 2004. Effective elastic properties of alumina-zirconia composite ceramics-Part 4. Tensile modulus of porous alumina and zirconia. Ceramics-Silikáty 48 (4), 165-174.

Peelen, J., Rejda, B., de Groot, K., 1978. Preparation and properties of sintered hydroxylapatite. Ceramurgia International 4 (2), 71-74.

Phani, K., 1986. Young's modulus-porosity relation in gypsum systems. American Ceramic Society Bulletin 65, 1584-1586.

Pichler, B., Hellmich, C., 2010. Estimation of influence tensors for eigenstressed multiphase elastic media with nonaligned inclusion phases of arbitrary ellipsoidal shape. Journal of Engineering Mechanics 136 (8), 10431053.

Pichler, B., Hellmich, C., 2011. Upscaling quasi-brittle strength of cement paste and mortar: A multi-scale engineering mechanics model. Cement and Concrete Research 41 (5), 467-476.

Pichler, B., Hellmich, C., Eberhardsteiner, J., 2008. Spherical and acicular representation of hydrates in a micromechanical model for cement paste: prediction of early-age elasticity and strength. Acta Mechanica 203 (3), 137-162.

Pichler, B., Hellmich, C., Eberhardsteiner, J., Wasserbauer, J., Termkhajornkit, P., Barbarulo, R., Chanvillard, G., 2013. Effect of gel-space ratio and microstructure on strength of hydrating cementitious materials: An engineering micromechanics approach. Cement and Concrete Research 45, $55-68$.

Prostak, K., Lees, S., 1996. Visualization of crystal-matrix structure. In situ demineralization of mineralized turkey leg tendon and bone. Calcified Tissue International 59 (6), $474-479$. 
Qu, T., Verma, D., A. M., Tomar, V., 2015a. Influence of interfacial interactions on deformation mechanism and interface viscosity in $\alpha$-chitincalcite interfaces. Acta Biomaterialia 25, 325-338.

Qu, T., Verma, D., Shahidi, M., Pichler, B., Hellmich, C., Tomar, V., 2015b. Mechanics of organic-inorganic biointerfaces - Implications for strength and creep properties. MRS Bulletin 40 (4), 349-358.

Reynaud, C., Thévenot, F., Chartier, T., Besson, J.-L., 2005. Mechanical properties and mechanical behaviour of $\mathrm{SiC}$ dense-porous laminates. Journal of the European Ceramic Society 25 (45), 589-597.

Rousselier, G., Luo, M., 2014. A fully coupled void damage and MohrCoulomb based ductile fracture model in the framework of a reduced texture methodology. International Journal of Plasticity 55, 1-24.

Roussette, S., Michel, J., Suquet, P., 2009. Nonuniform transformation field analysis of elastic-viscoplastic composites. Composites Science and Technology 69 (1), 22-27.

Saka, H., Goto, D., Moon, W.-J., 2008. Dislocations in plastically deformed apatite. Journal of Materials Science 43 (9), 3234-3239.

Salençon, J., 2001. Handbook of continuum mechanics, 1st Edition. Springer, Heidelberg, Germany.

Sanahuja, J., Dormieux, L., Meille, S., Hellmich, C., Fritsch, A., 2010. Micromechanical explanation of elasticity and strength of gypsum: from elongated anisotropic crystals to isotropic porous polycrystals. Journal of Engineering Mechanics 136 (2), 239 - 253.

Schneider, P., Meier, M., Wepf, R., Müller, R., 2011. Serial FIB/SEM imaging for quantitative 3D assessment of the osteocyte lacuno-canalicular network. Bone 49 (2), 304-311.

Schneider, P., Stauber, M., Voide, R., Stampanoni, M., Donahue, L., Müller, R., 2007. Ultrastructural properties in cortical bone vary greatly in two inbred strains of mice as assessed by synchrotron light based micro- and nano-CT. Journal of Bone and Mineral Research 22 (10), 1557-1570. 
Schwiedrzik, J., Raghavan, R., Burki, A., LeNader, V., Wolfram, U., Michler, J., Zysset, P., 2014. In situ micro pillar compression reveals superior strength and ductility but an absence of damage in lamellar bone. Nature Materials 13, 740747.

Shahidi, M., Pichler, B., Hellmich, C., 2014. Viscous interfaces as source for material creep: A continuum micromechanics approach. European Journal of Mechanics - A/Solids 45, 41-58.

Shareef, M., Messer, P., van Noort, R., 1993. Fabrication, characterization and fracture study of a machinable hydroxyapatite ceramic. Biomaterials $14(1), 69-75$.

Shen, W., Oueslati, A., de Saxcé, G., 2015. Macroscopic criterion for ductile porous materials based on a statically admissible microscopic stress field. International Journal of Plasticity 70, 60-76.

Shen, W., Shao, J., 2016. An incremental micro-macro model for porous geomaterials with double porosity and inclusion. International Journal of Plasticity 83, 37-54.

Shen, W., Shao, J., Kondo, D., Gatmiri, B., 2012. A micro-macro model for clayey rocks with a plastic compressible porous matrix. International Journal of Plasticity 36, 64-85.

Shojaei, A., Li, G., 2013. Viscoplasticity analysis of semicrystalline polymers: A multiscale approach within micromechanics framework. International Journal of Plasticity 42, 31-49.

Simo, J.-C., Hughes, T. J.-R., 1998. Computational Inelasticity, 1st Edition. Springer, Heidelberg, Germany.

Simo, J.-C., Taylor, R.-L., 1985. Consistent tangent operators for rateindependent elastoplasticity. Computer Methods in Applied Mechanics and Engineering 48 (1), 101-118.

Skedros, J., Holmes, J., Vajda, E., Bloebaum, R., 2005. Cement lines of secondary osteons in human bone are not mineral-deficient: new data in a historical perspective. The Anatomical Record A 286 (1), 781-803. 
Sokoloff, L., 1973. A note on the histology of cement lines. In: Perspectives in Biomedical Engineering. Springer, pp. 135-138.

Stroud, A., 1971. Approximate calculation of multiple integrals. Prentice Hall, Englewood Cliffs, NJ.

Su, X., Sun, K., Cui, F., Landis, W., 2003. Organization of apatite crystals in human woven bone. Bone 32 (2), $150-162$.

Tazawa, E., 1998. Effect of self stress on flexural strength of gypsum-polymer composites. Advanced Cement Based Materials 7 (1), 1-7.

Vena, P., Gastaldi, D., Contro, R., 2007. Determination of the effective elastic-plastic response of metal-ceramic composites. International Journal of Plasticity 24 (3), 483-508.

Viswanath, B., Raghavan, R., Ramamurty, U., Ravishankar, N., 2007. Mechanical properties and anisotropy in hydroxyapatite single crystals. Scripta Materialia 57 (4), 361-364.

Vuong, J., Hellmich, C., 2011. Bone fibrillogenesis and mineralization: Quantitative analysis and implications for tissue elasticity. Journal of Theoretical Biology 287, 115-130.

Weidenreich, F., 1930. Das Knochengewebe. In: Handbuch der mikroskopischen Anatomie des Menschen. Springer, pp. 391-520.

Weiner, S., Wagner, H., 1998. The material bone: structure-mechanical function relations. Annual Review of Materials Science 48, 271-298.

Weinmann, J., Sicher, H., 1955. Bone and bones. Mosby, St. Louis.

Wenk, H.-R., Voltolini, M., Mazurek, M., Van Loon, L. R., Vinsot, A., 2008. Preferred orientations and anisotropy in shales: Callovo-oxfordian shale (France) and opalinus clay (Switzerland). Clays and Clay Minerals 56 (3), $285-306$.

Zaoui, A., 2002. Continuum micromechanics: Survey. Journal of Engineering Mechanics 128 (8), 808-816. 
Zeng, T., Shao, J., Xu, W., 2014. Multiscale modeling of cohesive geomaterials with a polycrystalline approach. Mechanics of Materials 69 (1), 132 145.

Zylberberg, L., Traub, W., de Buffrenil, V., Allizard, F., T., A., Weiner, S., 1998. Rostrum of a toothed whale: ultrastructural study of a very dense bone. Bone 23, 241-247. 